\title{
Experimental Manipulation of Phosphoinositide lipids, from cells to organisms
}

\author{
Mehdi Doumane, Marie-Cécile Caillaud*, Yvon Jaillais*. \\ Laboratoire Reproduction et Développement des Plantes (RDP), Université de Lyon, ENS de Lyon, CNRS, INRAE, F-69342 \\ Lyon, France. \\ * Corresponding authors: yvon.jaillais@ens-lyon.fr , marie-cécile.caillaud@ens-lyon.fr
}

\section{keywords}

Optogenetics, lipid, membrane, targeted manipulation, model organism

\begin{abstract} can affect every aspect of membrane biology, including protein function (e.g., recruitment, activity), membrane physicochemical properties (e.g., curvature, surface charges, packing), and the generation of second messengers. PIs act at very precise locations within the cell in a dosedependent manner, and their local concentration can vary drastically during signaling and trafficking. Techniques able to manipulate PI amounts acutely and with subcellular accuracy are thus paramount to understand the role of these lipids in vivo. Here, we review these methods and emphasize the approaches recently developed to perturb PI levels in multicellular organisms.
\end{abstract}

Phosphoinositides (PIs) constitute a dynamic and interlinked network of landmark lipids in cellular membranes

PIs are phosphorylated derivatives from the glycerophospholipid phosphatidylinositol (PtdIns) [1]. The inositol head group can be phosphorylated in position three, four or five, forming up to seven distinct species of phosphorylated PtdIns, collectively known as phosphoinositides (PIs): PtdIns3P, PtdIns4P, PtdIns5P; PtdIns(3,4) $\mathrm{P}_{2}, \quad \operatorname{PtdIns}(3,5) \mathrm{P}_{2}, \quad \operatorname{PtdIns}(4,5) \mathrm{P}_{2}$ and PtdIns $(3,4,5) \mathrm{P}_{3}$ (Figure 1). Of note, the kinases that phosphorylate $\operatorname{PtdIns}(4,5) \mathrm{P}_{2}$ on the $3^{\text {rd }}$ position are only present in animals and as a result $\mathrm{PI}(3,4) \mathrm{P}_{2}$ and $\mathrm{PI}(3,4,5,) \mathrm{P}_{3}$ are not detected in fungal and plant cell membranes [2].

Each PI species localizes in specific organelles [3]. PI differential accumulation in membranes is critical for many cellular processes, and requires various cascading enzymatic activities to interconvert PIs into one another (Figure 1) [1, 4, 5]. PI kinases and phosphatases or phospholipases have various substrate and catalytic activities, subcellular localizations, spatiotemporal expression patterns and control mechanisms. Thus, PI-related enzymes (Glossary Box) constitute an extensive toolkit that precisely define PI concentrations and localization. Most of these enzymes act in a non-redundant manner at the cellular and organismal levels, although they may carry similar catalytic activities [1,6]. Additionally, longterm PI modifications may lead to indirect and pleiotropic effects that can be difficult to interpret because PIs: (i) regulate many cellular processes; (ii) are present in multiple cellular compartments at the same time; and (iii) are highly interdependent on each other. Furthermore, depleted enzymes might have functions beyond their catalytic activity, making it even more hazardous to conclude that the observed effects originate from the modulation of the PI species 
itself. Thus, even though knockout and knockdown strategies for depleting the PI levels are very useful, inducible and targeted methods are essential to untangle the direct and indirect function of PIs. Deciphering the direct impacts of PIs in cells and how their perturbation leads to complex phenotypes and diseases remains one of the most pressing issues in lipid biology and membrane signaling.

Here, we review the diverse methodologies used to manipulate PI levels in cells and organisms and discuss their respective advantages and limitations. We consider the pharmacological approaches developed to inhibit PI-related enzymes. We address how the inhibitions of PIrelated enzymes are applied to disturb the flux within the PI network and to assess the function of each PI species. We also examine how lipid analogs, including caged lipids, have recently been harnessed to heighten the concentration of a given PI. Furthermore, we report on recent genetic strategies established to modify PIs in an inducible and targeted manner, featuring chemogenetics, optogenetics and voltogenetics approaches. We discuss their optimal implementation for in vitro studies in cell lines and for experiments with animal and plant models. We describe how PI-related enzymes have been repurposed and engineered to build complex inducible systems, with ever-increasing spatiotemporal resolution and tailored specificities. Finally, as key take-home messages, we provide examples on how these arrays of experimental techniques can be used to tackle the complex functions of PIs in cells and organismal development and physiology.

\section{Pharmacological approaches for PI manipulation}

It is rather intuitive that, to modulate the respective levels of PIs, drugs preventing their synthesis or their hydrolysis would be tools of choice. Figure 1 recapitulates some of the compounds used to perturb the PI network. Most are chemicals targeting PI kinases (in particular PtdIns 3-kinases and PtdIns 4-kinases which are high-value targets in oncology, immunology and virology). These compounds curb the production of a given PI species while it is still consumed by PI phosphatases and phospholipases [7, 8]. PI levels can also be adjusted through inhibition or activation of phosphatases and phospholipases that hydrolyze them (Figure 1). For example, several inhibitors of the Phosphatase and TENsin homolog (PTEN) and Src Homology2-containing 5-phosphatase (SHIP) directly modulate the pool of PtdIns $(3,4,5) \mathrm{P}_{3}$ (Figure 1) $[9,10]$. Note that relatively few compounds can affect the levels of PtdIns $(4,5) \mathrm{P}_{2}$ by either targeting PtdIns4P 5-kinases or PtdIns(4,5) $\mathrm{P}_{2}$ 5-phosphatases (Figure 1), despite the importance of this lipid in cell biology.

PI manipulation via small molecules has been used in isolated cells, in plant and animal models and in clinical set-ups [7, 8, 10-13]. However, as tools to address fundamental questions about PI function, they have some limitations: (i) they are only active within a specific concentration range; (ii) they may have off-target effects; (iii) they may not penetrate easily in complex tissues; and (iv) they impact the whole sample and cannot target precise tissues, cells or organelles. Nonetheless, pharmacological modulation of PI-related enzymes constitutes a relative fast and easy modification of PI levels without the use of complex experimental and genetic systems.

\section{Delivery of PIs and PI analogues}

Modulation of PI levels can also be achieved by directly increasing the number of PI molecules. PIs are highly anionic and, as such, are largely impermeable to membranes when added exogenously. To tackle this issue, membrane permeable PI analogs have been developed (Figure 2A, [14-16]). The negative charges of these lipids are neutralized by protective groups, making them membrane permeable. After entering the cell, endogenous cytosolic esterases 
hydrolyze the chemical groups, exposing the functional inositol head. Delivery of PIs and PI analogs showed that PtdIns $(3,4) \mathrm{P}_{2}$ and $\mathrm{PtdIns}(3,4,5) \mathrm{P}_{2}$ stimulate clathrin-mediated endocytosis, tyrosine kinase receptor recycling and cell protrusion in animal cultured cells [17-19]. Injection of PtdIns $(4,5) \mathrm{P}_{2}$ analogs in Drosophila embryos undergoing cellularization, increased the GFP$\mathrm{PH}^{\mathrm{PLC}}$ PtdIns $(4,5) \mathrm{P}_{2}$ biosensor signal at the plasma membranes, illustrating that the method efficiently elevated PtdIns(4,5) $\mathrm{P}_{2}$ levels [20]. In these conditions, myosin-II was more stable at the plasma membrane, suggesting a role of $\operatorname{PtdIns}(4,5) \mathrm{P}_{2}$ in myosin membrane association in vivo [20]. Non-modified (as well as fluorescent) PIs were also successfully provided to plant cells, including Arabidopsis seedlings, using polyamine carriers neutralizing the negative charges of PI phosphate groups [21-23]. Delivery of $\operatorname{PtdIns}(3,5) \mathrm{P}_{2}$ led to vacuolar defects mimicking the phenotype observed upon loss-of-function of the putative 5-phosphatase AtSAC2-AtSAC5 [21].

When interpreting results from PI delivery assays, it is critical to remember that exogenously added lipids may artificially concentrate in non-endogenous compartments or on the wrong membrane leaflet. They may also be rapidly modified into another lipid species by endogenous enzymes. A solution to these problems is the use of caged-PI analogs that can be acutely activated (Figure 2B) [24-26]. In that case, the PI head is protected by a photoremovable group, which allows light-regulated uncaging at specific organelles [25, 27]. Membrane permeable, photoactivatable PtdIns $(4,5) \mathrm{P}_{2}$ can be loaded into cells in an inactive form and subsequently uncaged by light (Figure 2B). Such photoactivation is extremely fast (i.e., less than a second) and thus allow manipulating $\operatorname{PtdIns}(4,5) \mathrm{P}_{2}$ levels faster than its metabolism. Caged PtdIns $(4,5) \mathrm{P}_{2}$ were used to study the role of this lipid in exocytosis in mouse adrenal chromaffin cells [24].

Taken together, PI analogs are valuable chemicals, that can even be suitable for studies in multicellular organisms. However, lipid analogs remain expensive and their synthesis requires specialized skills, which limits the widespread use of this technique [25, 28]. Genetically encoded strategies for PI perturbation complement the approaches mentioned above. They rely on stable or transitory transfer of genetic constructs that, when expressed by host cells, will perturb PI homeostasis [28, 29]. Among these strategies, we will distinguish chronic from acute modulation techniques.

\section{Constitutively expressed genetic systems for PI manipulation}

Overexpression of genetic constructs that encode PI kinases, phosphatases or phospholipases, allows the chronic modification of PI levels (Figure 3A). However, the constitutive and ubiquitous perturbation of PIs is rarely viable in multicellular organisms. To by-pass lethality, it is possible to express the enzyme in specific tissues. For example, $\operatorname{PtdIns}(4,5) \mathrm{P}_{2}$ depletion in Drosophila spermatocytes was achieved by expressing the Salmonella PtdIns $(4,5) \mathrm{P}_{2}$ phosphatase SigD in this cell type [30]. Furthermore, the overexpression of the PtdIns4P 5kinase Skittles specifically in the germ line led to the opposite phenotypes compared to those induced by SigD ectopic expression [30]. Constitutive manipulation of PIs in few cell types avoids potential problems due to lethality and therefore allows studying the roles of PIs in cells that are in a physiological context.

It is also possible to repurpose PI modifying enzymes to localize their enzymatic activities to specific membrane compartments (Figure 3B). For instance, the endogenous PtdIns4P phosphatase called Sac1 normally resides in the membrane of the Endoplasmic Reticulum (ER). However, by taking the isolated phosphatase domain of Sacl and targeting it to the plasma membrane via a membrane anchor (e.g., Myristoylation And Palmitoylation - MAP), it is 
possible to rewire the Sac1 function to specifically erase PtdIns4P at the plasma membrane, not at the ER (Figure 3B). Such rewiring approach was used in transiently transformed Nicotiana benthamiana plants [31]. Upon expression of MAP-Sac1, PtdIns4P biosensors are solubilized from the plasma membrane and delocalized to intracellular compartments, indicating a decrease of the PtdIns4P pool at the plasma membrane [31]. Sac1-mediated PtdIns4P depletion in transient assays showed that PtdIns4P is the main anionic lipid responsible for the membrane electrostatic field in plant cells $[31,32]$ and that it is required for the plasma membrane association of many plant proteins [33-36].

\section{Inducible expression of PI-related enzymes}

As an alternative to the constitutive expression, it is also possible to use inducible expression systems. For example, estradiol-inducible expression of Arabidopsis PtdIns4P 5-Kinases $(P I P 5 K s)$ (induction for $24 \mathrm{~h}$ ) allowed to study the developmental impact of their overexpression and particularly their role in polar auxin transport and root gravitropism [37, 38]. However, by using endogenous enzymes, it is difficult to know whether the phenotypes are caused by the overexpression of the enzyme itself or the accumulation of its product (i.e., PtdIns $\left.(4,5) \mathrm{P}_{2}\right)$. To circumvent this pitfall, Gujas et al., exploited a genetic approach that enabled

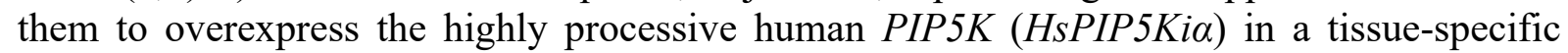
inducible manner. GFP-HsPIP5Ki $\alpha$ could be detected only three hours post induction, and PtdIns4P and PtdIns(4,5) $\mathrm{P}_{2}$ levels were already affected one hour after induction [39]. This approach revealed that ectopic PtdIns(4,5) $\mathrm{P}_{2}$ production impacted the differentiation of plant vascular tissues. A similar strategy was also used for the inducible depletion of PtdIns $(4,5) \mathrm{P}_{2}$ in plant cells (Figure 3C) [40]. The system is based on the expression of the catalytic domain of Drosophila $O C R L$ (encoding a PtdIns(4,5) $\mathrm{P}_{2}$ 5-phosphatase), which unlike human OCRL is active at $20^{\circ} \mathrm{C}$, fused to a membrane targeting sequence (i.e., MAP) and to a red fluorescent protein. Using a suite of genetic control, this tool was shown to specifically targets PtdIns(4,5) $\mathrm{P}_{2}$ without massively influencing the other anionic lipids present at the plasma membrane. The synthetic enzyme was detected three hours after the gene induction and $\operatorname{PtdIns}(4,5) \mathrm{P}_{2}$ levels already decreased approximately one hour post induction $[39,40]$. This strategy was deployed to study the impact of PtdIns(4,5) $\mathrm{P}_{2}$ depletion on plant root and shoot development and on the localization of plasma membrane proteins involved in endocytosis and cytoskeleton dynamics [40].

Altogether, these studies highlight that inducible genetic approaches are useful to address the developmental impact of lipid modification in multicellular organisms. They act in the range of a few hours, which is still slow compared to the speed of the endogenous PI metabolism. Alternative assays allow acute PI modification and rely on genetically encoded systems that can be tuned by a chemical (chemogenetics), by light (optogenetics) or by voltage (voltogenetics) [29].

\section{Acute pharmacological induction of endogenous PI metabolic enzymes}

PIs are known for their roles as secondary messengers. In particular, $\operatorname{PtdIns}(4,5) \mathrm{P}_{2}$ is hydrolyzed by Phospholipase C (PLC) into Diacylglycerols (DAG) and Inositol 1,4,5trisphosphate $\operatorname{Ins}(1,4,5) \mathrm{P}_{3}$ and both act as secondary messengers, triggering $\mathrm{Ca}^{2+}$ influx in the cytoplasm and modulating $\mathrm{K}^{+}$channels conductivity. PLCs also breakdown other PI species to a lesser extent. PLC-mediated hydrolysis of PtdIns(4,5) $\mathrm{P}_{2}$ occurs in response to (nor)epinephrine or acetylcholine perception by G-Protein Coupled Receptor (GPCR) (Figure 4A, [41]). It is possible to take advantage of these signaling pathways by stimulating them, thus acutely depleting plasma membrane PIs. Many studies used cell types that did not express the 
corresponding receptors, and therefore the cells had to be transfected with the signaling components $[42,43]$. In vitro mammalian cells transfected with the muscarinic acetylcholine receptor $\mathrm{M} 1\left(\mathrm{M}_{1} \mathrm{R}\right)$ and stimulated with the acetylcholine agonist oxotremorine $\mathrm{M}$ (Oxo-M) for $60 \mathrm{~s}$ showed a decreased PI level by roughly $70 \%$ [44]. PtdIns(4,5) $\mathrm{P}_{2}$ depletion was observed at $20 \mathrm{~s}$ post activation using the $\mathrm{PH}^{\mathrm{PLC}}$ fluorescent reporter solubilization or resonant energy transfer biosensors read-outs [45, 46]. Oxo-M-triggered $\operatorname{PtdIns}(4,5) \mathrm{P}_{2}$ perturbation substantiated the function of OCRL in dephosphorylating $\operatorname{PtdIns}(4,5) \mathrm{P}_{2}$ on endocytic vesicles [47]. Relying on the $M_{1} R$ receptor has the advantage of being very rapid and of exploiting endogenous components. However, the $\mathrm{M}_{1} \mathrm{R}$ signaling pathway itself regulates endogenous cellular processes, which must be considered when interpreting results from PLC-induced depletion of PIs.

\section{Chemogenetics: chemically-induced genetic strategies for acute manipulation of PIs}

The rapamycin-inducible method allows to acutely recruit lipid modifying enzymes from the cytosol to a given membrane within few seconds [28]. It is based on the inducible heterodimerization of the FKB domain of the mammalian Target of Rapamycin (mTOR) protein with FKBP12. One of the components (e.g., FRB domain) is anchored to a specific organelle via a membrane targeting sequence, while the other (e.g., FKBP domain) is fused to the catalytic domain of a PI-related enzyme [48-51]. Because the second construct consists only of the isolated catalytic domain, its default localization is in the cytoplasm. When it is not attached to a membrane, the enzymatic module has a low activity, which is likely due to poor access to its lipid substrate. However, a treatment with rapamycin induces the recruitment of the chimeric construct to its target membrane, allowing the enzyme to meet its substrate and thus to almost simultaneously catalyze the reaction. Using a rapamycin-inducible system, a 5phosphatase domain can associate with the plasma membrane in $\sim 30 \mathrm{~s}$, and $\operatorname{PtdIns}(4,5) \mathrm{P}_{2}$ biosensors are concomitantly solubilized, indicating acute $\operatorname{PtdIns}(4,5) \mathrm{P}_{2}$ depletion (Figure 4B, [49-51]). Alternatively, the 3-phosphatase MTM1 can be recruited to Rab5-containing early endosomes to specifically deplete PtdIns3P from the membrane of this compartment [48]. Since the first publications of this lipid modifying approach [48-51], the method was rewired to modify virtually all PI species through the recruitment of phosphatases, phospholipases or kinases at different subcellular localization (see Table 1). For example, concomitant rapamycin-inducible depletion of plasma membrane PtdIns $(4,5) \mathrm{P}_{2}$ and $\operatorname{PtdIns} 4 \mathrm{P}$ showed that PtdIns4P, together with PtdIns $(4,5) \mathrm{P}_{2}$, contributes to the electrostatic field at the surface of animal cell plasma membrane and to the localization of the polarity protein DISCS LARGE (Dlg) in Drosophila [52, 53]. This system was also utilized to challenge the presence and the function of various subcellular pools of PIs, including PtdIns, PtdIns4P, PtdIns( 3,4$) \mathrm{P}_{2}$ and PtdIns(3,5) $\mathrm{P}_{2}$ [54-60]. In addition, rapamycin-inducible dimerization was employed to probe the importance of PIs in membrane contact sites structure-function relationship. In particular, this strategy allowed asking whether the PtdIns4P phosphatase Sac1 acts in cis (i.e., at the ER membrane) or in trans (i.e., at the plasma membrane or in the trans-Golgi Network) in animal cells [61, 62]. FRB/FKBP-mediated accumulation of $\operatorname{PtdIns}(3,4,5) \mathrm{P}_{3}$ was reported in Drosophila larva, proving that the approach was viable for PI manipulation in multicellular organisms [63]. This method was also recently implemented in plants using transient assays in N. Benthamiana for the inducible depletion of PtdIns4P at the plasma membrane [64].

The FRB/FKBP system shows acute manipulation of PIs in membranes of in vitro animal cell lines but also in plants using transient assays. The FRB/FKBP strategy has, however, some limitations, including the irreversibility of dimerization induced by rapamycin. This drawback can be overcome by the addition of a competitive ligand [65]. Also, rapamycin treatment interferes with endogenous mTOR complexes that have pleiotropic functions. To avoid this 
issue, rapamycin analogs that do not target endogenous mTOR proteins [66] and controls strategies have two components and thus require the genetic expression of two constructs simultaneously. Expressing proteins from several plasmids is not always easy, especially when working with cells or organisms that are difficult to transform and can lead to heterogeneous accumulation levels. To circumvent these difficulties, it is possible to express both parts on a single construct (e.g. by using self-cleaving peptides) [67]. Alternatively, single-component chemogenetic designs are emerging that are based on the so-called self-localizing ligandinduced protein translocation (SLIPT) strategy [28, 68-70]. In SLIPT, a ligand is engineered to accumulate at a specific subcellular localization and then it recruits a protein of interest (e.g., PI-related enzyme) (Figure 4C).

\section{Optogenetics: light-induced genetic systems for acute manipulation of PIs}

Optogenetic lipid modification approaches resemble rapamycin-inducible methods in the sense that they often rely on inducible dimerization strategies, aiming at rewiring subcellular localization of PI-related enzymes. However, in the case of optogenetics, specific wavelengths of light trigger heterodimerization between a photoreceptor and its downstream partner. Most lipid-modifying optogenetic techniques exploit plant or bacterial blue-light photoreceptors (e.g., cryptochrome, LOV-domain) (Figure 5A and Table 1) [29, 71-75]. It is also possible to use phytochrome-based strategies [76]. Phytochromes have the advantage to induce protein dimerization in response to red light, with far-red light reversing the reaction, therefore acting like an active ON-OFF toggle switch (Figure 5B). Each photoreceptor has distinct sensitivity and kinetics of dimerization; thus, they can be engineered to achieve various optogenetic properties in terms of speed of action, reversibility and responsiveness [77]. Like the rapamycin-inducible method, it is now possible to manipulate the levels of many PI species using various enzymatic modules at different intracellular membranes (see Table 1).

The advantages of the optogenetic strategies include: (i) no treatment with potentially toxic molecules; (ii) no small molecule diffusion into the medium and inside the cell; (iii) their speed of action; (iv) their reversibility; and (v) the ability to pattern light for stimulation of the optogenetic tool in a group of cells or subcellular compartments. Furthermore, using optogenetics, it is possible to obtain subcellular precision by targeting one of the components to specific organelles (see Table 1); and through photomanipulation. For instance, actuation of CRY2-OCRL by an evanescent blue light wave, generated at the coverslip, can selectively perturb the pool of PtdIns $(4,5) \mathrm{P}_{2}$ located at the plasma membrane contacting the coverslip [71]. Also, a blue light pulse on a region of the plasma membrane can manipulate PI concentration locally $[71,78]$. On the other end, like for chemogenetics, optogenetic approaches often require the genetic expression of two constructs simultaneously. However, a system dubbed optoPB, based on a blue-light-sensitive LOV domain enables inducible translocation to the plasma membrane with a single engineered protein [74]. In optoPB, blue light induces a conformational change that exposes a polybasic tail $[74,79]$. The polybasic tail in turn interacts with the negatively charged plasma membrane, thereby triggering cytosol-to-plasma membrane relocalization using a single chimeric construct (Figure 5C).

Optogenetics strategies have limitations to consider. First, optogenetic systems are induced by light; thus, they may require dedicated equipment, such as a green room to manipulate the samples under non-inducible lights. Patterning light in a rapid and reproducible manner under the microscope may also require dedicated hardware for photomanipulation [76]. Second, the use of the optogenetic constructs limits the number of channels that are available for imaging. Indeed, if possible, it is best to avoid fluorescent proteins that are excited by the same 
wavelength as the photoreceptor present in the optogenetic actuator. Third, photoreceptors in most living organisms (e.g., flavin-based chromophores used by blue-light photoreceptors). In that case, the availability of the chromophore is not an issue. The presence of the chromophore may, however, be limiting for phytochrome-based strategies. Indeed, phytochromes require a light-sensing bilin chromophore, which is not produced by animal cells. The chromophores thus need to be added exogenously before the optogenetic experiments [76]. Bilin chromophores, such as phycocyanobilin, are relatively unstable, and their injection in living metazoans can be inefficient (e.g., poor or no diffusion to the target tissues) and unreliable (e.g., rapid degradation). By contrast, bilin chromophores are endogenously synthesized in plants. However, the development of optogenetics in the green lineage is challenging, because photosynthetic organisms need light to survive.

\section{Voltogenetics: voltage-induced genetic approaches for acute manipulation of PIs}

The discovery of voltage-dependent PI phosphatases, the so-called VSPs, led to the development of voltage-controlled and genetically encoded (voltogenetics) tools for PI acute manipulation [80-82]. Wild-type Ci-VSP and Dr-VSP are mainly PtdIns $(4,5) \mathrm{P}_{2}$ 5-phosphatases and are found in Ciona intestinalis (Metazoa Urochordata) and zebrafish (Danio rerio, Metazoa Teleostei), respectively. Upon transfection into human cultured cells, a $\sim 1 \mathrm{~s}$ depolarization (from - $20 \mathrm{mV}$ to $+100 \mathrm{mV}$ ) transiently erases $\operatorname{PtdIns}(4,5) \mathrm{P}_{2}$ [83]. Interestingly, the initial PtdIns $(4,5) \mathrm{P}_{2}$ levels are recovered $\sim 15 \mathrm{~s}$ after repolarization. Using a voltogenetic approach in Xenopus oocytes, it was recently confirmed that the activity of $\mathrm{K}^{+}$channel Slo3 is PtdIns(4,5) $\mathrm{P}_{2}$-sensitive [84]. VSPs can also be engineered to accommodate additional enzymatic activities. The Ci-VSP voltage-sensitive domain (VSD) was fused to PTEN (CiVSPTEN), which specifically modifies the pool of $\operatorname{PtdIns}(3,4,5) \mathrm{P}_{3}$ and $\operatorname{PtdIns}(3,4) \mathrm{P}_{2}$ in response to electric stimulation in vivo [85]. Electrophysiology approaches require specific expertise, and may be difficult to apply to live organisms, but they are fast and reversible and may be used to assess in vivo phosphatase activity upon heterologous expression.

\section{Concluding Remarks}

In this review, we highlighted various experimental systems deployed to manipulate PIs in different models. These techniques include pharmacological and genetic strategies as well as acute and chronic modifications. We can distinguish approaches that allow rapid and highly targeted manipulations but that are mainly used in cultured cells, from slower perturbation strategies that are more amenable to multicellular organisms such as animals and plants. Researchers should thus choose the methods according to their questions and biological models. Addressing the role of PIs in morphogenesis may not need ultrafast modifications, because of the dynamics of development by comparison to that of PI metabolism. By opposition, the study of very fast processes, such as vesicular trafficking or membrane contact establishment may require the implementation of rapid perturbation techniques. Optogenetics appears as a particularly promising strategy that should allow bridging the gap between research in isolated cells and in whole organisms (outstanding question box). One can envision that light-mediated fast lipid perturbations may soon allow analyzing the role of PIs over a large range of time scales. It may then be possible to address both the direct molecular targets of PIs and their downstream developmental or physiological effects. 
Glossary box

PI-related enzyme: Enzyme that impacts PI metabolism or dynamics, and that includes PI kinases, phosphatases or phospholipases.

PI biosensors: Transgenic expression of lipid-binding domains that interact with a given PI species in a stereospecific manner, fused with a fluorescent protein (i.e., genetically encoded sensors). Such probe works as a translocation sensor, because they translocate from the cytosol to a given membrane upon interaction with their cognate lipid and they dissociate from that membrane upon removal of the PI species.

Resonant Energy Transfer biosensors: Intramolecular biosensors that change conformation upon binding to a given PI species. The change of conformation is detected by a change in the fluorescence resonant energy transfer (FRET) or bioluminescence resonant energy transfer (BRET) that happens when the distance between the two fluorescent proteins (a donor and an acceptor) present in the biosensor varies. Resonant energy transfer biosensors can detect dynamic and rapid variations in PI levels and are more quantitative than translocation sensors.

Caged PIs: Chemically modified lipids that renders the PI head group mainly inert until it is removed for example using light.

Chemogenetics: Genetically-encoded system that allows manipulating a biological pathway using small molecules.

Optogenetics: Genetically-encoded system that allows manipulating a biological pathway using light. Voltogenetics: Genetically-encoded system that allows manipulating a biological pathway using electric stimulation.

Secondary messenger: Signaling molecules released in the cell in response to a primary signal and that trigger signal transduction cascade(s).

Rapamycin: Small molecule inhibitor of mTOR derived from Streptomyces hygroscopicus, that has potent antiproliferative and immunosuppressant properties.

Photomanipulation: Describes a range of techniques that allow microscopists to target illumination to specific areas of interests in their field of view, e.g., to photoactivate, photobleach or photoablate a given protein, organelle, cells or group of cells.

Inducible expression system: genetically encoded systems that enable researchers to induce the expression of a gene in response to a given stimulus (e.g., small molecule, light).

Myristoylation: Non-reversible lipid modification used to anchor proteins into biological membranes, via the covalent attachment of a myristoyl group to an $\mathrm{N}$-terminal Glycine.

Palmitoylation: Reversible lipid modification of a protein, which promotes membrane association through S-acylation of cysteine residues (e.g., covalent attachment of palmitic acid or other type of fatty acids).

Photoreceptor: Protein specialized in detecting certain wavelengths of light and to transduce this information into specific signals, often via conformational changes. Most photoreceptors function in conjunction with light-sensitive organic molecules known as chromophores.

Cryptochrome2 (CRY2): blue light photoreceptor from plants that uses Flavin adenine dinucleotide (FAD) as a light-harvesting chromophore. Blue light perception triggers the interaction with the Nterminus of the Arabidopsis thaliana transcription factor CIB1 (i.e. CIBN).

Phytochrome b (phyB): Plant protein covalently linked to a light-sensing bilin chromophore, that is sensitive to red and far-red light. Red light triggers the interaction with Phytochrome Interacting Factors (PIF) and far-red light their dissociation.

Light-Oxygen-Voltage-sensing (LOV) domain: Protein domain, present in plants, algae, as well as some bacteria and fungi, that in conjunction with a flavin mononucleotide chromophore acts as a bluelight photoreceptor.

\section{Acknowledgements}

We are grateful to the SiCE group (RDP, Lyon, France) for comments and discussions. YJ has received funding from the European Research Council (ERC) under the European Union's Horizon 2020 research and innovation program (Grant Agreement No 101001097), ANR caLIPSO (ANR-18-CE13-0025-02; YJ) and ANR STAYING-TIGHT (ANR-18-CE13-0016- 
02), MCC has received funding from ANRJC/JC JUNIOR INVESTIGATOR GRANT (ANR16-CE13-0021), SEED FUND ENS LYON-2016 and 2020, ANR PlantScape (ANR-20-CE130026-02) and ANR DIVCON (ANR-21-CE13-0016-03). MD was funded by Ph.D. fellowship from the French Ministry of Research and Higher Education.

The authors declare no competing interests.

\section{References}

1. Balla, T. (2013) Phosphoinositides: tiny lipids with giant impact on cell regulation. Physiol Rev 93 (3), 1019-137.

2. Platre, M.P. and Jaillais, Y. (2016) Guidelines for the Use of Protein Domains in Acidic Phospholipid Imaging. Methods Mol Biol 1376, 175-94.

3. Kutateladze, T.G. (2010) Translation of the phosphoinositide code by PI effectors. Nat Chem Biol 6 (7), 507-13.

4. Noack, L.C. and Jaillais, Y. (2020) Functions of Anionic Lipids in Plants. Annu Rev Plant Biol 71, 71102.

401 5. Noack, L.C. and Jaillais, Y. (2017) Precision targeting by phosphoinoistides: how PIs direct endomembrane trafficking in plants. . Current Opinion in Plant Biology 40.

6. Colin, L.A. and Jaillais, Y. (2019) Phospholipids across scales: lipid patterns and plant development. Curr Opin Plant Biol 53, 1-9.

7. Vanhaesebroeck, B. et al. (2021) PI3K inhibitors are finally coming of age. Nat Rev Drug Discov.

8. Li, Y.P. et al. (2021) Research progress of phosphatidylinositol 4-kinase and its inhibitors in inflammatory diseases. Eur J Pharmacol 907, 174300.

9. Kerr, W.G. et al. (2020) Small molecule targeting of SHIP1 and SHIP2. Biochemical Society Transactions 48 (1), 291-300.

10. Spinelli, L. et al. (2015) PTEN inhibitors: an evaluation of current compounds. Adv Biol Regul 57, 10211.

11. Ikonomov, O.C. et al. (2019) Small molecule PIKfyve inhibitors as cancer therapeutics: Translational promises and limitations. Toxicol Appl Pharmacol 383, 114771.

12. Sharma, G. et al. (2019) A family of PIKFYVE inhibitors with therapeutic potential against autophagydependent cancer cells disrupt multiple events in lysosome homeostasis. Autophagy 15 (10), 1694-1718.

13. Ronan, B. et al. (2014) A highly potent and selective Vps34 inhibitor alters vesicle trafficking and autophagy. Nat Chem Biol 10 (12), 1013-9.

14. Dinkel, C. et al. (2001) Membrane-Permeant 3-OH-Phosphorylated Phosphoinositide Derivatives. Angew Chem Int Ed Engl 40 (16), 3004-8.

15. Jiang, T. et al. (1998) Membrane-permeant esters of phosphatidylinositol 3,4,5-trisphosphate. J Biol Chem 273 (18), 11017-24.

16. Laketa, V. et al. (2009) Membrane-permeant phosphoinositide derivatives as modulators of growth factor signaling and neurite outgrowth. Chem Biol 16 (11), 1190-6.

17. Laketa, V. et al. (2014) $\mathrm{PIP}_{3}$ induces the recycling of receptor tyrosine kinases. Sci Signal 7 (308), ra5.

18. Posor, Y. et al. (2013) Spatiotemporal control of endocytosis by phosphatidylinositol-3,4-bisphosphate. Nature 499 (7457), 233-7.

19. Subramanian, D. et al. (2010) Activation of membrane-permeant caged PtdIns(3)P induces endosomal fusion in cells. Nat Chem Biol 6 (5), 324-6.

20. Reversi, A. et al. (2014) Plasma membrane phosphoinositide balance regulates cell shape during Drosophila embryo morphogenesis. J Cell Biol 205 (3), 395-408.

21. Novakova, P. et al. (2014) SAC phosphoinositide phosphatases at the tonoplast mediate vacuolar function in Arabidopsis. Proc Natl Acad Sci U S A 111 (7), 2818-23.

22. Mei, Y. et al. (2012) Arabidopsis phosphatidylinositol monophosphate 5-kinase 2 is involved in root gravitropism through regulation of polar auxin transport by affecting the cycling of PIN proteins. Cell Res 22 (3), 581-97.

23. Rubilar-Hernandez, C. et al. (2019) PI4KIIIbeta Activity Regulates Lateral Root Formation Driven by Endocytic Trafficking to the Vacuole. Plant Physiol 181 (1), 112-126. 
24. Walter, A.M. et al. (2017) Phosphatidylinositol 4,5-bisphosphate optical uncaging potentiates exocytosis. Elife 6.

25. Farley, S. et al. (2021) Caged lipids for subcellular manipulation. Curr Opin Chem Biol 65, 42-48.

26. Laguerre, A. and Schultz, C. (2018) Novel lipid tools and probes for biological investigations. Curr Opin Cell Biol 53, 97-104.

27. Flores, J. et al. (2020) Lipids: chemical tools for their synthesis, modification, and analysis. Chem Soc Rev 49 (14), 4602-4614.

28. Tei, R. and Baskin, J.M. (2021) Induced proximity tools for precise manipulation of lipid signaling. Curr Opin Chem Biol 65, 93-100.

29. Idevall-Hagren, O. and De Camilli, P. (2015) Detection and manipulation of phosphoinositides. Biochimica et Biophysica Acta (BBA) - Molecular and Cell Biology of Lipids 1851 (6), 736-745.

30. Gupta, A. et al. (2018) Phosphatidylinositol 4,5-bisphosphate regulates cilium transition zone maturation in Drosophila melanogaster. J Cell Sci 131 (16).

31. Simon, M.L. et al. (2016) A PtdIns(4)P-driven electrostatic field controls cell membrane identity and signalling in plants. Nat Plants 2, 16089.

32. Platre, M.P. et al. (2018) A Combinatorial Lipid Code Shapes the Electrostatic Landscape of Plant Endomembranes. Dev Cell 45 (4), 465-480 e11.

33. Ruiz-Lopez, N. et al. (2021) Synaptotagmins at the endoplasmic reticulum-plasma membrane contact sites maintain diacylglycerol homeostasis during abiotic stress. Plant Cell.

34. Doumane, M. and Caillaud, M.C. (2020) Assessing Extrinsic Membrane Protein Dependency to PI4P Using a Plasma Membrane to Endosome Relocalization Transient Assay in Nicotiana benthamiana. Methods Mol Biol 2177, 95-108.

35. Saile, S.C. et al. (2021) Arabidopsis ADR1 helper NLR immune receptors localize and function at the plasma membrane in a phospholipid dependent manner. New Phytol 232 (6), 2440-2456.

36. Reuter, L. et al. (2021) Light-triggered and phosphorylation-dependent 14-3-3 association with NONPHOTOTROPIC HYPOCOTYL 3 is required for hypocotyl phototropism. Nat Commun 12 (1), 6128.

37. Ischebeck, T. et al. (2013) Phosphatidylinositol 4,5-bisphosphate influences PIN polarization by controlling clathrin-mediated membrane trafficking in Arabidopsis. Plant Cell 25 (12), 4894-911.

38. Armengot, L. et al. (2016) Regulation of polar auxin transport by protein and lipid kinases. J Exp Bot 67 (14), 4015-37.

39. Gujas, B. et al. (2017) Perturbing phosphoinositide homeostasis oppositely affects vascular differentiation in Arabidopsis thaliana roots. Development 144 (19), 3578-3589.

40. Doumane, M. et al. (2021) Inducible depletion of PI(4,5)P2 by the synthetic iDePP system in Arabidopsis. Nat Plants 7 (5), 587-597.

41. Bill, C.A. and Vines, C.M. (2020) Phospholipase C. Adv Exp Med Biol 1131, 215-242.

42. Suh, B.C. et al. (2004) Regulation of KCNQ2/KCNQ3 current by G protein cycling: the kinetics of receptor-mediated signaling by Gq. J Gen Physiol 123 (6), 663-83.

43. Gulyás, G. et al. (2020) ORP3 phosphorylation regulates phosphatidylinositol 4-phosphate and $\mathrm{Ca}(2+)$ dynamics at plasma membrane-ER contact sites. J Cell Sci 133 (6).

44. Dickson, E.J. et al. (2016) Dynamic formation of ER-PM junctions presents a lipid phosphatase to regulate phosphoinositides. J Cell Biol 213 (1), 33-48.

45. Dong, R. et al. (2016) Endosome-ER Contacts Control Actin Nucleation and Retromer Function through VAP-Dependent Regulation of PI4P. Cell 166 (2), 408-23.

46. Myeong, J. et al. (2021) Compartmentalization of phosphatidylinositol 4,5-bisphosphate metabolism into plasma membrane liquid-ordered/raft domains. Proc Natl Acad Sci U S A 118 (9).

47. Nández, R. et al. (2014) A role of OCRL in clathrin-coated pit dynamics and uncoating revealed by studies of Lowe syndrome cells. Elife 3, e02975.

48. Fili, N. et al. (2006) Compartmental signal modulation: Endosomal phosphatidylinositol 3-phosphate controls endosome morphology and selective cargo sorting. Proc Natl Acad Sci U S A 103 (42), 15473-8.

49. Varnai, P. et al. (2006) Rapidly inducible changes in phosphatidylinositol 4,5-bisphosphate levels influence multiple regulatory functions of the lipid in intact living cells. Journal of Cell Biology 175 (3), 377382.

50. Suh, B.-C. et al. (2006) Rapid Chemically Induced Changes of PtdIns $(4,5) \mathrm{P}<\operatorname{sub}>2</$ sub $>$ Gate KCNQ Ion Channels. Science 314 (5804), 1454-1457.

51. Heo, W.D. et al. (2006) PI(3,4,5)P3 and PI(4,5)P2 lipids target proteins with polybasic clusters to the plasma membrane. Science 314 (5804), 1458-61. 
52. Dong, W. et al. (2015) A conserved polybasic domain mediates plasma membrane targeting of Lgl and its regulation by hypoxia. J Cell Biol 211 (2), 273-86.

53. Hammond, G.R. et al. (2012) PI4P and PI(4,5)P2 are essential but independent lipid determinants of membrane identity. Science 337 (6095), 727-30.

54. Zewe, J.P. et al. (2020) Probing the subcellular distribution of phosphatidylinositol reveals a surprising lack at the plasma membrane. J Cell Biol 219 (3).

55. Goulden, B.D. et al. (2019) A high-avidity biosensor reveals plasma membrane $\mathrm{PI}(3,4) \mathrm{P}(2)$ is predominantly a class I PI3K signaling product. J Cell Biol 218 (3), 1066-1079.

56. Hammond, G.R. et al. (2015) The ML1Nx2 Phosphatidylinositol 3,5-Bisphosphate Probe Shows Poor Selectivity in Cells. PLoS One 10 (10), e0139957.

57. Hammond, G.R. et al. (2014) A novel probe for phosphatidylinositol 4-phosphate reveals multiple pools beyond the Golgi. J Cell Biol 205 (1), 113-26.

58. Pemberton, J.G. et al. (2020) Defining the subcellular distribution and metabolic channeling of phosphatidylinositol. J Cell Biol 219 (3).

59. Szentpetery, Z. et al. (2010) Acute manipulation of Golgi phosphoinositides to assess their importance in cellular trafficking and signaling. Proc Natl Acad Sci U S A 107 (18), 8225-30.

60. Levin, R. et al. (2017) Multiphasic dynamics of phosphatidylinositol 4-phosphate during phagocytosis. Mol Biol Cell 28 (1), 128-140.

61. Zewe, J.P. et al. (2018) SAC1 degrades its lipid substrate PtdIns4P in the endoplasmic reticulum to maintain a steep chemical gradient with donor membranes. Elife 7.

62. Venditti, R. et al. (2019) The activity of Sac1 across ER-TGN contact sites requires the four-phosphateadaptor-protein-1. J Cell Biol 218 (3), 783-797.

63. Khuong, T.M. et al. (2013) Synaptic PI(3,4,5)P3 is required for Syntaxin1A clustering and neurotransmitter release. Neuron 77 (6), 1097-108.

64. Winkler, J. et al. (2021) Visualizing protein-protein interactions in plants by rapamycin-dependent delocalization. Plant Cell 33 (4), 1101-1117.

65. Feng, S. et al. (2014) A rapidly reversible chemical dimerizer system to study lipid signaling in living cells. Angew Chem Int Ed Engl 53 (26), 6720-3.

66. Bayle, J.H. et al. (2006) Rapamycin analogs with differential binding specificity permit orthogonal control of protein activity. Chem Biol 13 (1), 99-107.

67. Tóth, D.J. et al. (2012) Acute depletion of plasma membrane phosphatidylinositol 4,5-bisphosphate impairs specific steps in endocytosis of the G-protein-coupled receptor. J Cell Sci 125 (Pt 9), 2185-97.

68. Ishida, M. et al. (2013) Synthetic self-localizing ligands that control the spatial location of proteins in living cells. J Am Chem Soc 135 (34), 12684-9.

69. Nakamura, A. et al. (2020) Engineering Orthogonal, Plasma Membrane-Specific SLIPT Systems for Multiplexed Chemical Control of Signaling Pathways in Living Single Cells. ACS Chem Biol 15 (4), 10041015.

70. Grant, B.M.M. et al. (2020) A Non-Canonical Calmodulin Target Motif Comprising a Polybasic Region and Lipidated Terminal Residue Regulates Localization. Int J Mol Sci 21 (8).

71. Idevall-Hagren, O. et al. (2012) Optogenetic control of phosphoinositide metabolism. Proc Natl Acad Sci U S A 109 (35), E2316-23.

72. Benedetti, L. et al. (2020) Optimized Vivid-derived Magnets photodimerizers for subcellular optogenetics in mammalian cells. Elife 9.

73. Benedetti, L. et al. (2018) Light-activated protein interaction with high spatial subcellular confinement. Proceedings of the National Academy of Sciences 115 (10), E2238-E2245.

74. He, L. et al. (2017) Optical control of membrane tethering and interorganellar communication at nanoscales. Chem Sci 8 (8), 5275-5281.

75. Kawano, F. et al. (2015) Engineered pairs of distinct photoswitches for optogenetic control of cellular proteins. Nat Commun 6, 6256.

76. Toettcher, J.E. et al. (2011) Light-based feedback for controlling intracellular signaling dynamics. Nat Methods 8 (10), 837-9.

77. Manoilov, K.Y. et al. (2021) A guide to the optogenetic regulation of endogenous molecules. Nat Methods 18 (9), 1027-1037.

78. Giordano, F. et al. (2013) PI(4,5)P(2)-dependent and Ca(2+)-regulated ER-PM interactions mediated by the extended synaptotagmins. Cell 153 (7), 1494-509. 
79. Li, L. et al. (2021) Structural Determinants for Light-Dependent Membrane Binding of a Photoswitchable Polybasic Domain. ACS Synth Biol 10 (3), 542-551.

80. Ratzan, W.J. et al. (2011) Voltage sensitive phosphoinositide phosphatases of Xenopus: their tissue distribution and voltage dependence. J Cell Physiol 226 (11), 2740-6.

81. Kawanabe, A. et al. (2020) Engineering an enhanced voltage-sensing phosphatase. J Gen Physiol 152 (5).

82. Okamura, Y. et al. (2018) Voltage-Sensing Phosphatases: Biophysics, Physiology, and Molecular Engineering. Physiol Rev 98 (4), 2097-2131.

83. Falkenburger, B.H. et al. (2010) Kinetics of PIP2 metabolism and KCNQ2/3 channel regulation studied with a voltage-sensitive phosphatase in living cells. J Gen Physiol 135 (2), 99-114.

84. Kawai, T. and Okamura, Y. (2020) The Slo3/Lrrc52 complex is sensitive to phosphoinositides. Channels (Austin) 14 (1), 1-3.

85. Lacroix, J. et al. (2011) Controlling the activity of a phosphatase and tensin homolog (PTEN) by membrane potential. J Biol Chem 286 (20), 17945-53.

86. Inoue, T. et al. (2005) An inducible translocation strategy to rapidly activate and inhibit small GTPase signaling pathways. Nat Methods 2 (6), 415-8.

87. Bisaria, A. et al. (2020) Membrane-proximal F-actin restricts local membrane protrusions and directs cell migration. Science 368 (6496), 1205-1210.

88. Putyrski, M. and Schultz, C. (2011) Switching heterotrimeric G protein subunits with a chemical dimerizer. Chem Biol 18 (9), 1126-33.

89. van Unen, J. et al. (2015) Plasma membrane restricted RhoGEF activity is sufficient for RhoA-mediated actin polymerization. Sci Rep 5, 14693.

90. Komatsu, T. et al. (2010) Organelle-specific, rapid induction of molecular activities and membrane tethering. Nat Methods 7 (3), 206-8.

91. Robinson, M.S. et al. (2010) Rapid inactivation of proteins by rapamycin-induced rerouting to mitochondria. Dev Cell 18 (2), 324-31.

92. Cheeseman, L.P. et al. (2013) Specific removal of TACC3-ch-TOG-clathrin at metaphase deregulates kinetochore fiber tension. J Cell Sci 126 (Pt 9), 2102-13.

93. Xie, B. et al. (2016) Plasma Membrane Phosphatidylinositol 4,5-Bisphosphate Regulates Ca(2+)-Influx and Insulin Secretion from Pancreatic $\beta$ Cells. Cell Chem Biol 23 (7), 816-826.

94. Kennedy, M.J. et al. (2010) Rapid blue-light-mediated induction of protein interactions in living cells. Nat Methods 7 (12), 973-5.

95. Valon, L. et al. (2017) Optogenetic control of cellular forces and mechanotransduction. Nat Commun 8, 14396.

96. Nguyen, P.M. et al. (2019) The PI(4)P phosphatase Sac2 controls insulin granule docking and release. J Cell Biol 218 (11), 3714-3729.

97. O'Neill, P.R. et al. (2016) Subcellular optogenetic activation of Cdc42 controls local and distal signaling to drive immune cell migration. Mol Biol Cell 27 (9), 1442-50.

98. Natwick, D.E. and Collins, S.R. (2021) Optimized iLID Membrane Anchors for Local Optogenetic Protein Recruitment. ACS Synth Biol 10 (5), 1009-1023. 99. Guntas, G. et al. (2015) Engineering an improved light-induced dimer (iLID) for controlling the localization and activity of signaling proteins. Proc Natl Acad Sci U S A 112 (1), 112-7. 


\section{Highlights:}

- Phosphoinositides (PIs) are signaling lipids because they are precursors of second messengers or variations in their local quantity act as a signal in itself.

- PI metabolism is fast and interdependent as they are rapidly converted into each other by kinases and phosphatases.

- Mutations of PI enzymes impacts development, but it is difficult to untangle the direct from indirect phenotypes induced by chronic PI perturbations.

- Experimental systems can acutely manipulate PI levels with subcellular accuracy in cultured cells. These include chemogenetic, voltogenetic and optogenetic strategies and operate in relevant time frames that are faster than the endogenous PI metabolism.

- Systems for the inducible manipulation of PIs are becoming available in multicellular organisms, which enable to address the function of these lipids during development and in their in vivo physiological context. 


\section{Outstanding question box}

- Will it possible to manipulate PI levels on demand by complementing the palette of available PI-related enzyme inhibitors? In particular, we urgently need to uncover pan-PIP5K drugs to prevent PtdIns $(4,5) \mathrm{P}_{2}$ synthesis and to characterize additional PI phosphatase inhibitors, notably to target the SAC and INPP protein families.

- To what extent is it still feasible to improve the diversity of PIs that can be labeled, caged and delivered into cells? We should also strive to make these approaches more widely available to the research community, perhaps by using in cellulo tagging strategies.

- Minor pools of lipids can be difficult to visualize because of their tiny concentrations, but they may nonetheless be highly relevant in terms of function and physiology. Can acute perturbation methods be leveraged to uncover the roles of minor pools of specific PI species?

- Can we engineer synthetic manipulation systems to experimentally perturb lipid homeostasis at the nanoscale? Such assay may allow uncoupling the function of different lipid pools that are present on the same membrane and could help to probe PI dynamics and diffusion.

- Most optogenetic lipid modification strategies are based on inducible dimerization approaches. Future research should aim at engineering new light-gated modules to directly manipulate the activity of PI-related enzymes.

- What are the technical limitations in designing acute perturbation systems that are compatible with studies in multicellular organisms and to tackle their roles in development, normal physiology, and disease progression?

- How important is the fatty acid chain composition in PI function in vivo? This has remained mainly unanswered and we should design experimental methods to probe and perturb PI fatty acid composition in vivo. 


\begin{tabular}{|c|c|c|c|c|}
\hline $\begin{array}{l}\text { Type of inducible } \\
\text { systema }^{a}\end{array}$ & Construct $^{b}$ & Reaction/ targeting $^{\mathrm{C}}$ & Refs & Addgene \# \\
\hline \multirow{29}{*}{$\begin{array}{l}\text { Rapamycin } \\
\text { (or rapalog)-inducible } \\
\text { dimerization }\end{array}$} & FKBP-PI4KA ${ }^{\Delta N}$ & Ptdlns $\rightarrow$ Ptdlns4P & {$[57]$} & 139311 \\
\hline & FKBP-PI4KB & Ptdlns $\rightarrow$ Ptdlns4P & [54] & 139310 \\
\hline & FKBP-PIP5K & Ptdlns4P $\rightarrow$ Ptdlns $(4,5) \mathrm{P}_{2}$ & {$[50]$} & 20157 \\
\hline & FKBP-iSH2 & Ptdlns $(4,5) \mathrm{P}_{2} \rightarrow$ Ptdlns $(3,4,5) \mathrm{P}_{3}$ & {$[50]$} & 20159 \\
\hline & FKBP-PTEN & Ptdlns $(3,4,5) \mathrm{P}_{3} \rightarrow$ Ptdlns $(4,5) \mathrm{P}_{2}$ & [55] & 116866 \\
\hline & FKBP-PLC $\delta 1^{\Delta 44-\Delta \mathrm{PH}}$ & Ptdlns $(4,5) \mathrm{P}_{2} \rightarrow \operatorname{lns}(1,4,5) \mathrm{P}_{3}$ and DAG & [58] & - \\
\hline & FKBP-PJ & Ptdlns $(4,5) \mathrm{P}_{2} \rightarrow$ Ptdlns4P $\rightarrow$ Ptdlns & [53] & 37999 \\
\hline & FKBP-Inp54p & Ptdlns $(4,5) \mathrm{P}_{2} \rightarrow$ Ptdlns $4 \mathrm{P}$ & $\begin{array}{l}{[50,} \\
51]\end{array}$ & 20155 \\
\hline & FKBP-INPP5E & Ptdlns $(4,5) \mathrm{P}_{2} \rightarrow$ Ptdlns4P & [49] & 67516 \\
\hline & FKBP-PJINPP5E & Ptdlns $(4,5) \mathrm{P}_{2} \rightarrow$ Ptdlns4P & [53] & 38001 \\
\hline & FKBP-PJSAC & Ptdlns4P $\rightarrow$ Ptdlns & {$[53]$} & 38000 \\
\hline & FKBP-Sac1 ${ }^{\Delta \mathrm{TDM}}$ & Ptdlns4P $\rightarrow$ Ptdlns & [61] & 108132 \\
\hline & $\mathrm{Sac1}^{\Delta \mathrm{TDM}}$-FKBP & Ptdlns4P $\rightarrow$ Ptdlns & [61] & 108123 \\
\hline & FKBP-BcPI-PLC ${ }^{A A}$ & Ptdlns $\rightarrow$ Ins1P and DAG & {$[58]$} & - \\
\hline & FKBP-INPP4B & Ptdlns $(3,4) \mathrm{P}_{2} \rightarrow$ Ptdlns3P & [55] & 116864 \\
\hline & FKBP-MTM1 & Ptdlns3P $\rightarrow$ Ptdlns & {$[57]$} & 51614 \\
\hline & Lyn $^{\mathrm{N} 11}-\mathrm{FRB}$ & Plasma membrane & $\begin{array}{l}{[49-51} \\
53,57 \\
86,87]\end{array}$ & $\begin{array}{l}20147, \\
67517, \\
38003, \\
38004, \\
155228 \\
\end{array}$ \\
\hline & GAP43 ${ }^{\mathrm{N19}}-\mathrm{FRB}$ & Plasma membrane & [49] & 67518 \\
\hline & Lck $^{\mathrm{N10}}-\mathrm{FRB}$ & Plasma membrane & $\begin{array}{l}{[88,} \\
89]\end{array}$ & 67902 \\
\hline & FRB-Sac1 ${ }^{\mathrm{C} 67}$ & Endoplasmic reticulum & [54] & 139317 \\
\hline & FRB-PMPC10 & Peroxisome & [54] & 13916 \\
\hline & FRB-Giantin & Golgi & $\begin{array}{l}{[54,} \\
89,90]\end{array}$ & $\begin{array}{l}139313, \\
67903 \\
\end{array}$ \\
\hline & FRB-Rab5 & Early endosome & {$[57]$} & $\begin{array}{l}51612 \\
64209\end{array}$ \\
\hline & FRB-Rab7 & Late endosome & {$[57]$} & $\begin{array}{l}51613 \\
64210 \\
\end{array}$ \\
\hline & Akap1 1 ${ }^{\mathrm{N} 31}$-FRB & Mitochondria & {$[54]$} & 139315 \\
\hline & Tom70p ${ }^{N 33}-F R B$ & Mitochondria & $\begin{array}{l}{[91,} \\
92]\end{array}$ & $\begin{array}{l}46942, \\
59352\end{array}$ \\
\hline & FRB-MoA ${ }^{N 37}$ & Mitonchondria & $\begin{array}{l}89, \\
90]\end{array}$ & 67904 \\
\hline & LAMP-FRB & Lysosome & $\begin{array}{l}\text { [55, } \\
90]\end{array}$ & - \\
\hline & TGN38-FRB & Trans-Golgi Network & [59] & - \\
\hline \multirow{10}{*}{$\begin{array}{l}\text { CRY2-based blue } \\
\text { light dimerization } \\
\text { system }\end{array}$} & CRY2-INPP5E & Ptdlns $(4,5) \mathrm{P}_{2} \rightarrow$ Ptdlns4P & [71] & 79561 \\
\hline & CRY2-OCRL & Ptdlns $(4,5) \mathrm{P}_{2} \rightarrow$ Ptdlns $4 \mathrm{P}$ & [71] & $\begin{array}{l}79566 \\
79565\end{array}$ \\
\hline & CRY2-iSH2 & Ptdlns $(4,5) \mathrm{P}_{2} \rightarrow \operatorname{Ptdlns}(3,4,5) \mathrm{P}_{3}$ & [71] & 66839 \\
\hline & CRY2-PIP5K1 & Ptdlns4P $\rightarrow$ Ptdlns $(4,5) \mathrm{P}_{2}$ & [93] & $\begin{array}{l}79569 \\
79570\end{array}$ \\
\hline & CIBN-Ras4BC14 & Plasma membrane & $\begin{array}{l}{[71,} \\
94]\end{array}$ & $\begin{array}{l}26867 \\
79574\end{array}$ \\
\hline & $\mathrm{Lyn}^{\mathrm{N} 11}-\mathrm{CIBN}$ & Plasma membrane & [71] & 79572 \\
\hline & ER-P450 C1 ${ }^{\mathrm{N} 37}$ & Endoplasmic reticulum & [73] & - \\
\hline & CIBN-OMP25 $5^{\mathrm{C} 44}$ & Mitochondria & [73] & - \\
\hline & CIBN-mitoNEET & Mitochondria & [95] & 89480 \\
\hline & Rab3-CIBN & Insulin granule & [96] & - \\
\hline $\begin{array}{l}\text { Magnet blue light } \\
\text { dimerization system }\end{array}$ & $\begin{array}{l}\text { pMagFast(3x)- } \\
\text { MTMR1 }\end{array}$ & Ptdlns3P $\rightarrow$ Ptdlns & [73] & - \\
\hline
\end{tabular}




\begin{tabular}{|c|c|c|c|c|}
\hline \multirow[t]{10}{*}{$\begin{array}{l}\text { (Neurospora crassa } \\
\text { LOVVIVID-based) }\end{array}$} & $\begin{array}{l}\text { pMagFast2(3x)- } \\
\text { OCRL }\end{array}$ & Ptdlns $(4,5) \mathrm{P}_{2} \rightarrow$ Ptdlns4P & [73] & - \\
\hline & $\begin{array}{l}\text { iSH2- } \\
\text { pMagFast2(3x) }\end{array}$ & Ptdlns $(4,5) \mathrm{P}_{2} \rightarrow$ Ptdlns $(3,4,5) \mathrm{P}_{3}$ & {$[75]$} & 67298 \\
\hline & iSH2-pMag(3x) & Ptdlns $(4,5) \mathrm{P}_{2} \rightarrow$ Ptdlns $(3,4,5) \mathrm{P}_{3}$ & [75] & 67304 \\
\hline & $\begin{array}{l}\text { nMag(1x)- } \\
\text { KRas4B }\end{array}$ & Plasma membrane & [73] & - \\
\hline & $\begin{array}{l}\text { nMag(3x)- } \\
\text { KRas4BC14 }\end{array}$ & Plasma membrane & [73] & - \\
\hline & P450 2C1 ${ }^{\text {N377}}$-nMag & Endoplasmic reticulum & [73] & - \\
\hline & $\begin{array}{l}\text { nMag(1x)- } \\
\text { OMP25 } 244\end{array}$ & Mitochondria & [73] & - \\
\hline & $\begin{array}{l}\text { nMag(2x)- } \\
\text { OMP25 }\end{array}$ & Mitochondria & [73] & - \\
\hline & Rab5-nMag & Early endosome & [73] & - \\
\hline & LAMTOR $^{\mathrm{N} 40}$-nMag & Lysosome & [73] & - \\
\hline \multirow{2}{*}{$\begin{array}{ll}\text { Enhanced } & \text { Magnet } \\
\text { dimerization } & \text { system } \\
\text { (thermostable) }\end{array}$} & eMagB ${ }^{F}-O C R L$ & Ptdlns $(4,5) \mathrm{P}_{2} \rightarrow$ Ptdlns4P & [72] & 162254 \\
\hline & eMag $^{F}-N-\operatorname{Ras}^{C}$ & Plasma membrane & [72] & 162247 \\
\hline \multirow{7}{*}{$\begin{array}{lr}\text { improved } & \text { Light } \\
\text { Dimerization } & \text { System } \\
- & \text { iLID } \\
\text { (Avena sativa } & \text { LOV2- } \\
\text { based blue } & \text { light } \\
\text { receptor) } & \end{array}$} & SspB R73Q-OCRL & Ptdlns $(4,5) \mathrm{P}_{2} \rightarrow$ Ptdlns4P & [73] & - \\
\hline & iLID-KRas4B ${ }^{\mathrm{C} 14}$ & Plasma membrane & $\begin{array}{l}{[87} \\
97-99]\end{array}$ & $\begin{array}{l}85680, \\
1600999, \\
604411, \\
155229 \\
\end{array}$ \\
\hline & Lyn $^{N 11}$-iLID & Plasma membrane & [98] & 161001 \\
\hline & ADRB2-iLID & $\begin{array}{l}\text { Plasma membrane } \\
\text { (slow diffusion) }\end{array}$ & [98] & 161002 \\
\hline & Stargazin-iLID & $\begin{array}{l}\text { Plasma membrane } \\
\text { (slow diffusion) }\end{array}$ & [98] & 161000 \\
\hline & P450 2C1 ${ }^{\text {N37-iLID }}$ & Endoplasmic reticulum & [73] & \\
\hline & iLID-ActA $^{\text {C46 }}$ & Mitochondria & [73] & $\begin{array}{l}60413 \\
60413 \\
\end{array}$ \\
\hline $\begin{array}{l}\text { Single component } \\
\text { LOV-based blue light } \\
\text { relocalization system }\end{array}$ & iSH2-optoPB & $\begin{array}{l}\text { Ptdlns }(4,5) \mathrm{P}_{2} \rightarrow \\
\text { at Plasma membrane }\end{array}$ & {$[74]$} & - \\
\hline \multirow{2}{*}{$\begin{array}{l}\text { Phytochrome-based } \\
\text { Red/Far Red } \\
\text { dimerization switch }\end{array}$} & iSH2-PIF & Ptdlns $(4,5) \mathrm{P}_{2} \rightarrow$ Ptdlns $(3,4,5) \mathrm{P}_{3}$ & {$[76]$} & 50841 \\
\hline & PhyB- K-Ras4B ${ }^{\mathrm{C} 14}$ & Plasma membrane & {$[76]$} & 50839 \\
\hline $\begin{array}{l}\text { Voltage-based } \\
\text { system (VSP) }\end{array}$ & CiDr-VSP L223F & $\begin{array}{l}\text { Plasma membrane Ptdlns }(4,5) \mathrm{P}_{2} \rightarrow \\
\text { Ptdlns4P }\end{array}$ & [81] & 140892 \\
\hline
\end{tabular}

Table 1. List of constructs used in chemogenetic, optogenetics and voltogenetic systems to acutely perturb PI levels at precise subcellular localization.

597 aTwo-component systems are grouped together with light color-shade indicating the enzyme 598 and dark color-shade indicating the membrane anchor.

$599{ }^{b}$ Controls consisting of catalytically dead enzyme exist for most systems but are not indicated 600 in this table for clarity purpose.

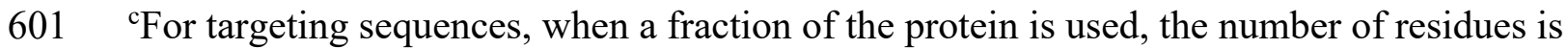
602 indicated and their location ( ${ }^{\mathrm{N}}$ for $\mathrm{N}$-terminus, ${ }^{\mathrm{C}}$ for $\mathrm{C}$-terminus) 


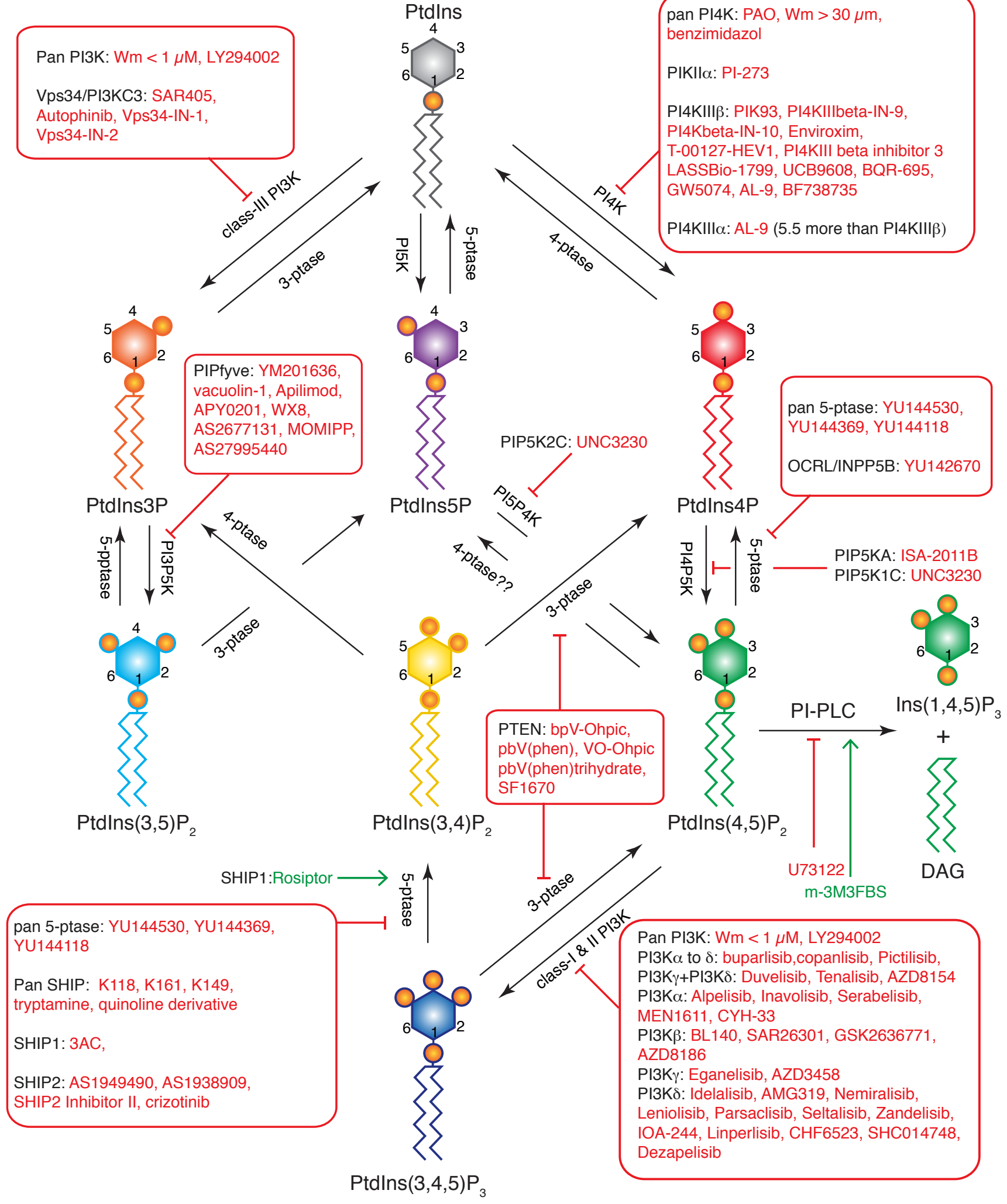


606 Figure 1. Small molecule perturbation of the PI network. Schematic representation of PI

607 molecules and their interconversion via kinases/phosphatases mediated by 608 phosphorylation/dephosphorylation cycles. Small molecules described to inhibit (in red) or 609 activate (in green) phosphorylation of dephosphorylation reaction. The enzyme (in human) 610 targeted by each compound are indicated in black, when known. Note that the list of small 611 molecule inhibitors may not be exhaustive and they may not always be fully specific of the 612 indicated enzyme and only show preferential activity toward them.

613 Key legend: PI, phosphoinositide; PtdIns, phosphatidylinositol; PtdIns3P, 614 phosphatidylinositol-3-phosphate; PtdIns4P, phosphatidylinositol-4-phosphate; PtdIns5P, 615 phosphatidylinositol-5-phosphate; $\operatorname{PtdIns}(3,4) \mathrm{P}_{2}$, phosphatidylinositol-(3,4)-bisphosphate; 616 PtdIns(4,5) $\mathrm{P}_{2}$, phosphatidylinositol-(4,5)-bisphosphate; PtdIns(3,5) $\mathrm{P}_{2}$, phosphatidylinositol617 (3,5)-bisphosphate; $\operatorname{PtdIns}(3,4,5) \mathrm{P}_{2}$, phosphatidylinositol-(3,4,5)-triphosphate; DAG, 618 diacylglycerol; Ins(1,4,5) $\mathrm{P}_{3}$; inositol-(1,3,5)-triphosphate; PI3K, PtdIns 3-Kinase; VPS34, 619 vacuolar protein sorting34; PI4K, PtdIns 4-Kinase; PI5K, PtdIns 5-Kinase; ptase; phosphatase; 620 PIKfyve, phosphoinositide kinase with a specificity for the five position containing a fyve 621 finger; OCRL, Lowe oculocerebrorenal syndrome protein; INPP5, Inositol polyphosphate 5622 phosphatase; PI3P5K; PtdIns3P 5-Kinase; PI5P4K; PtdIns5P 4-Kinase; PI4P5K; PtdIns4P 5623 Kinase; PI-PLC; phosphoinositide-specific phospholipase C; SHIP; Src homology 2 (SH2) 624 domain-containing inositol-5-phosphatase; Wm, wortmannin; PAO, phenylarsine oxide. 
(A) exogenous treatment

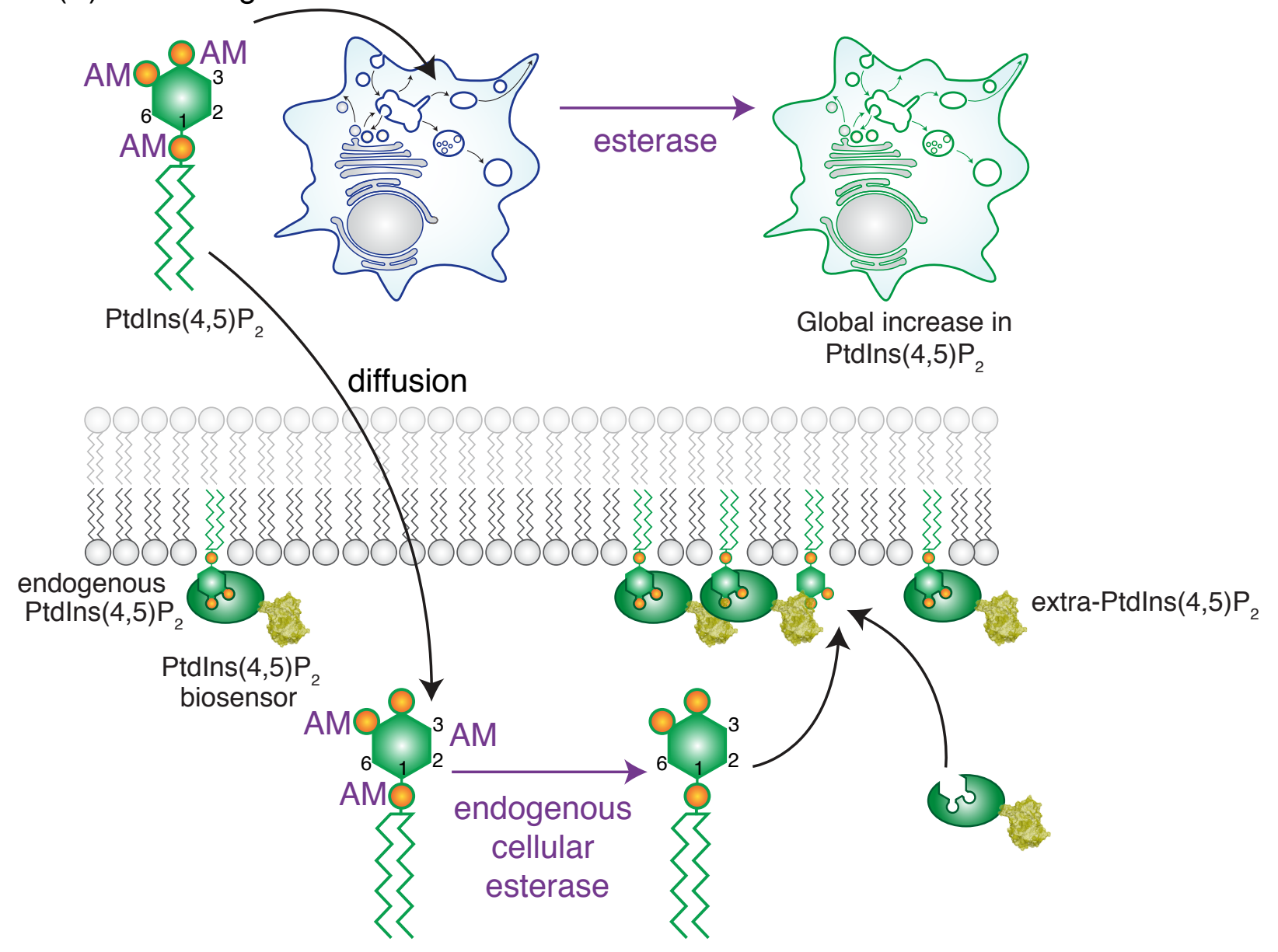

(B)

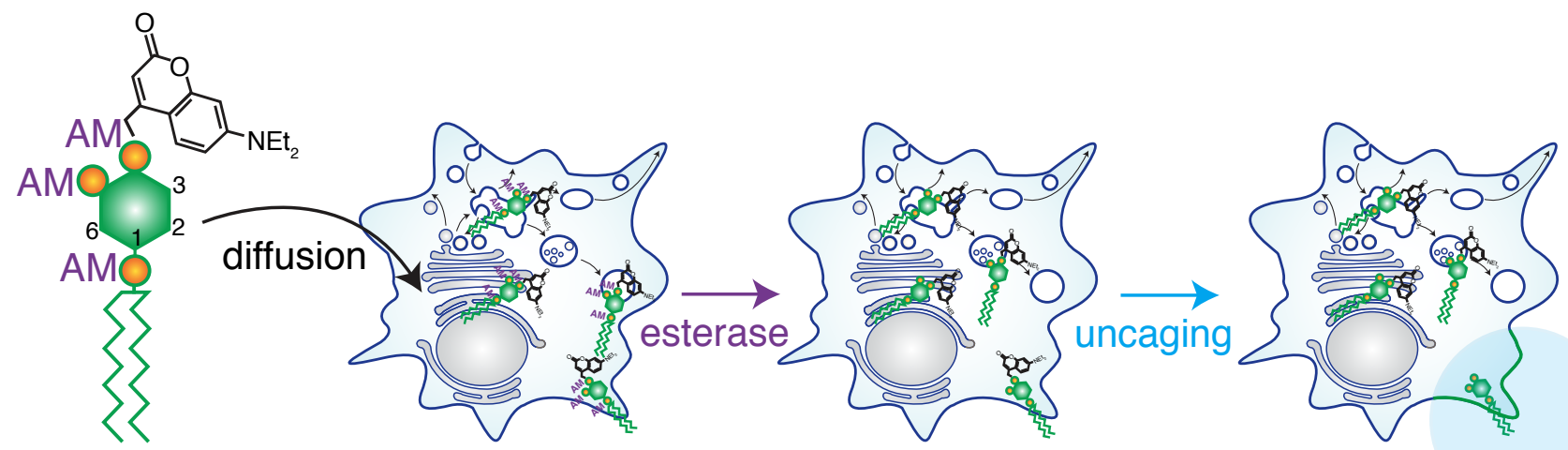

caged-Ptdlns $(4,5) \mathrm{P}_{2}$

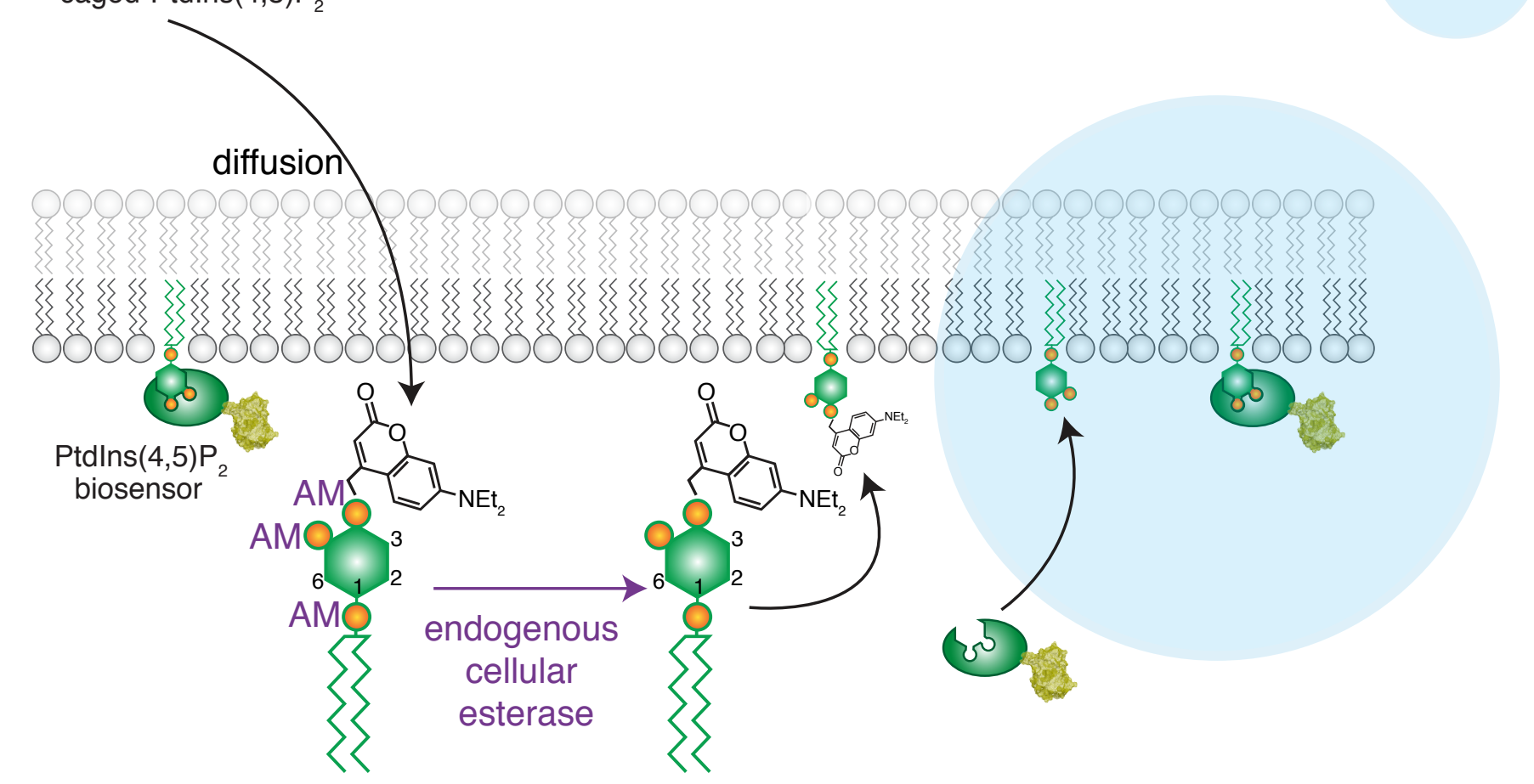


627 Figure 2. Strategy for the delivery of PI molecules into cells. A) Example showing the 628 delivery of membrane permeable PtdIns $(4,5) \mathrm{P}_{2}$ through the neutralization of the PI negative 629 charges via protective groups, such as acetoxymethyl (AM) esters. The protective groups are 630 subsequently removed inside the cell and the PI then becomes biologically active. B) Example 631 showing the delivery of caged $\operatorname{PtdIns}(4,5) \mathrm{P}_{2}$ into cells. These molecules are membrane 632 permeable and will become active upon blue light irradiation, which can be highly targeted 633 through the use of a directed light beam (i.e., photomanipulation).

634 Key legend: PI, phosphoinositide; PtdIns(4,5) $\mathrm{P}_{2}$, phosphatidylinositol-(4,5)-bisphosphate; 635 AM, acetoxymethyl esters. 
(A) Modulation of PI level via chronic over-expression of endogenous enzyme (e.g. PIP5K)

plasma membrane
(B)

$$
\begin{aligned}
& \text { Modulation of PI level via } \\
& \text { over-expression of synthetic enzyme } \\
& \text { (e.g. MAP-Sac1) }
\end{aligned}
$$

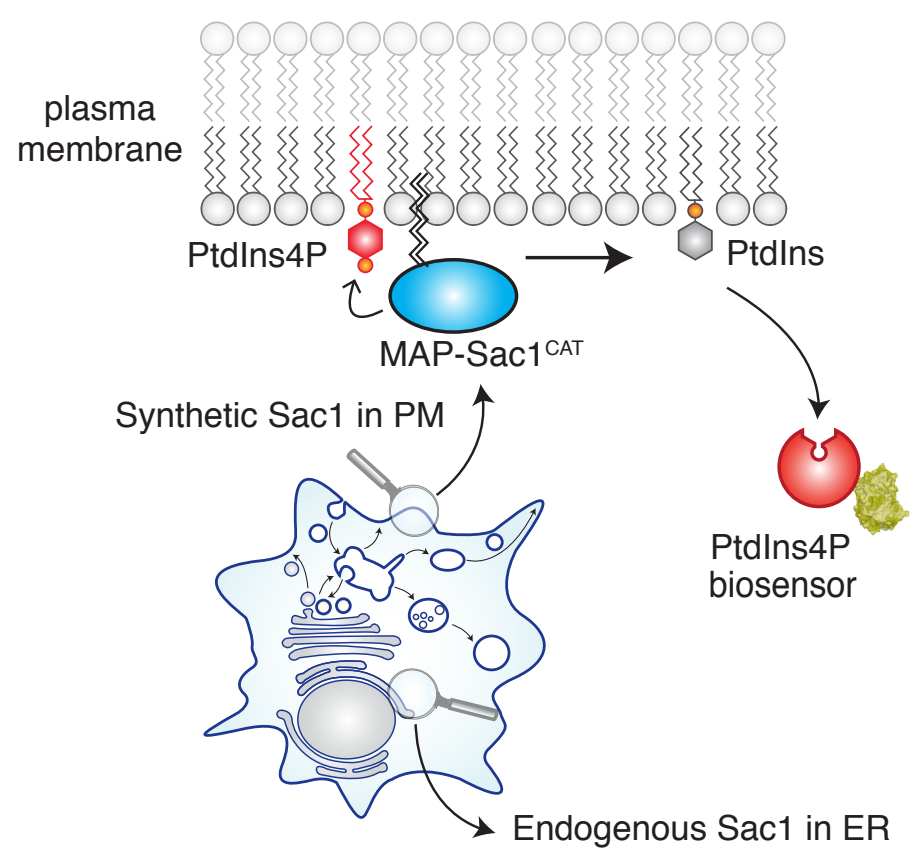

(C) Modulation of PI level via inducible over-expression of synthetic enzyme (e.g. MAP-OCRL)

no induction (90 min Mock)

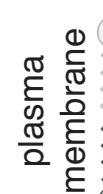

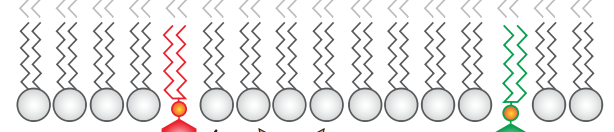

Ptdlns4P PIP5K

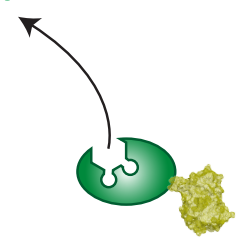

Ptdlns $(4,5) \mathrm{P}_{2}$ biosensor

induction (90 min DEX)

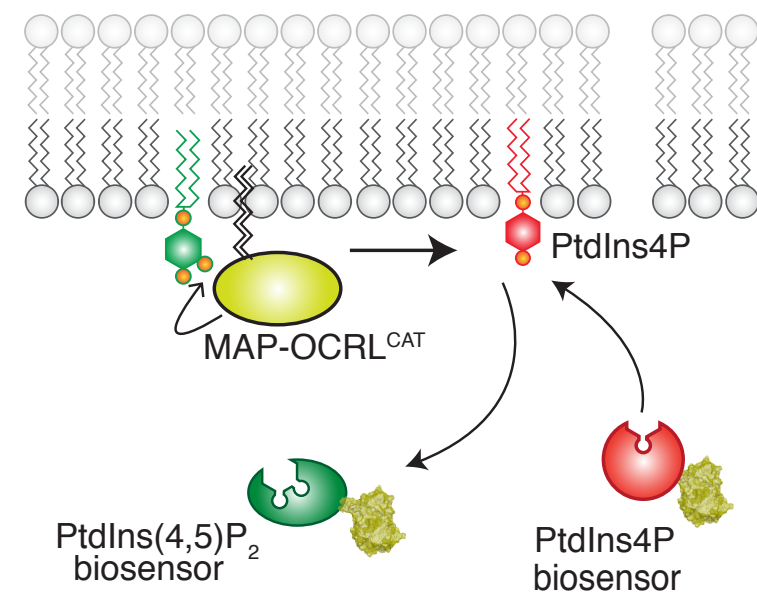

induction (90 min DEX)

\section{induction (90 min DEX)}

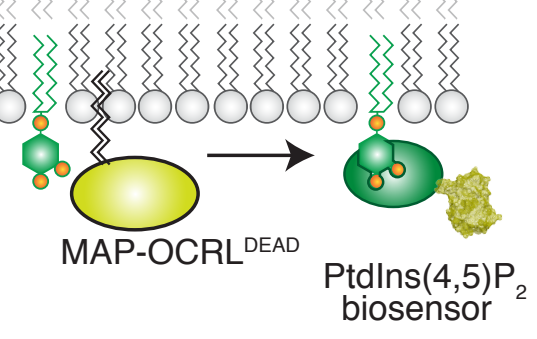

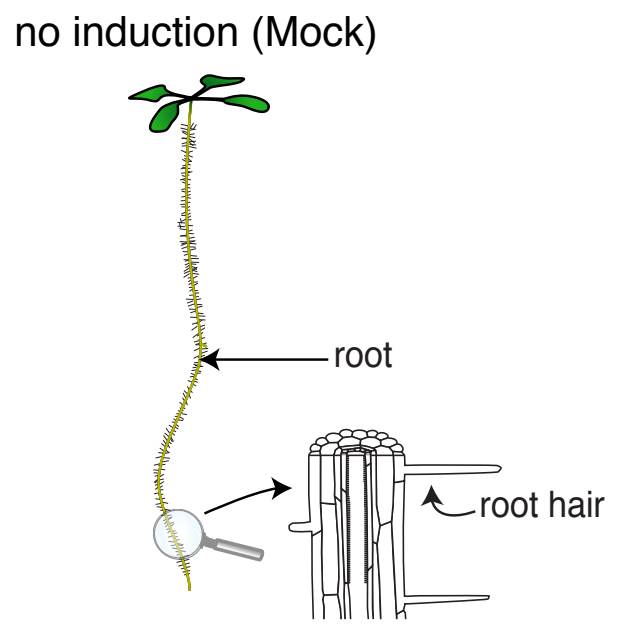

transfert $24 \mathrm{~h}$ on DEX

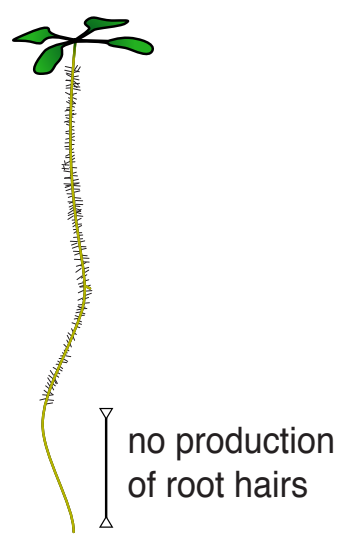

transfert $24 \mathrm{~h}$ on DEX

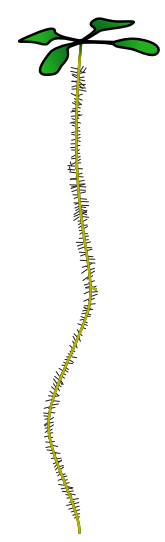

Ptdlns4P biosensor 
638 Figure 3. Manipulation of PIs via expression of wild type or engineered PI-related

639 enzymes. A) Example showing PI modification, here increased PtdIns $(4,5) \mathrm{P}_{2}$ synthesis, via the 640 chronic overexpression of a gene encoding a PI-related enzyme (e.g. PIP5K). B) Example 641 showing the engineering of PI-related enzyme to synthetically target a specific enzymatic 642 activity to a given subcellular localization. In this example, the endogenous Sac1 protein acts 643 at the membrane of the ER, but a synthetic Sac1 enzyme, consisting of only its catalytic domain 644 (Sac1 ${ }^{\mathrm{CAT}}$ ) and a plasma membrane targeting sequence (e.g. myristoylation and palmitoylation, 645 MAP), specifically targets the PtdIns4P pool at the cell surface. C) By coupling a synthetic 646 enzyme (as in B) and an inducible expression system (here using dexamethasone, DEX), it is 647 possible to obtain inducible PI modification to study their function during development. In this 648 example, a synthetic PtdIns(4,5) $\mathrm{P}_{2}$ phosphatase, consisting of the plasma membrane targeting 649 motif MAP and the 5-phosphatase OCRL from Drosophila efficiently perturb PtdIns $(4,5) \mathrm{P}_{2}$ 650 levels and can be used to study root development and morphogenesis in Arabidopsis (e.g. 651 formation of root hairs).

652 Key legend: PI, phosphoinositide; PtdIns, phosphatidylinositol; PtdIns4P,

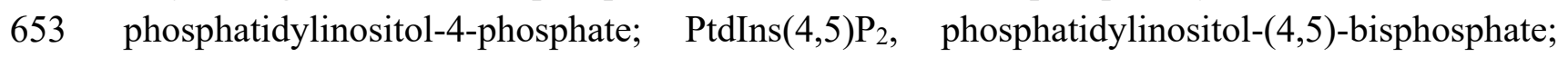
654 PIP5K; PtdIns4P 5-Kinase; MAP, myristoylation and palmitoylation; SAC, suppressor of actin; 655 PM, plasma membrane; ER, endoplasmic reticulum; DEX, dexamethasone; OCRL, Lowe 656 oculocerebrorenal syndrome protein; CAT, catalytic domain; DEAD, catalytically inactive. 
(A)

Acetylcholine/Oxo-M induced Ptdlns $(4,5) \mathrm{P}_{2}$ hydrolysis

acetylcholine / Oxo-M

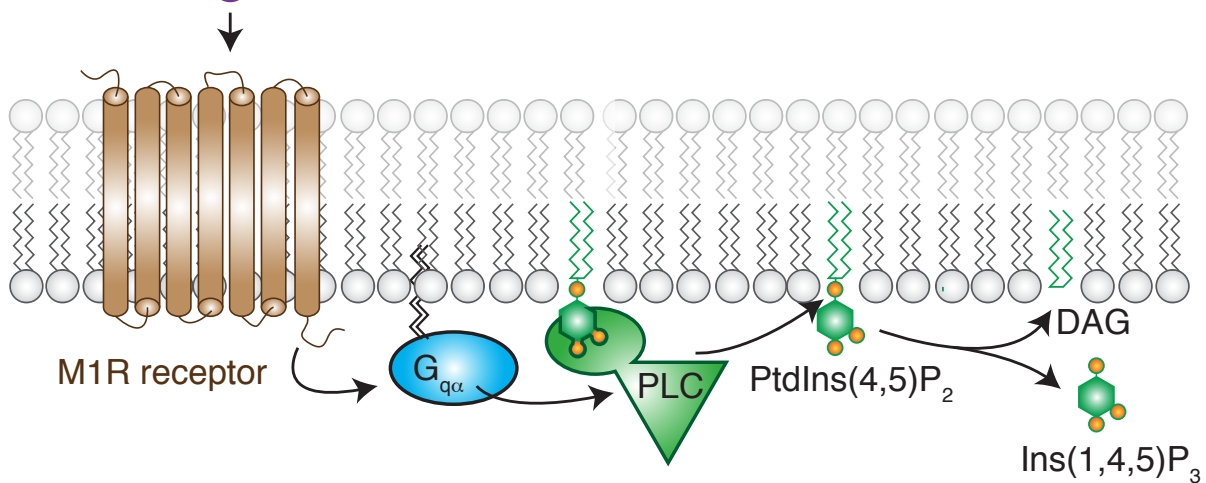

(B) Rapamycin-inducible relocalization system

rapamycin

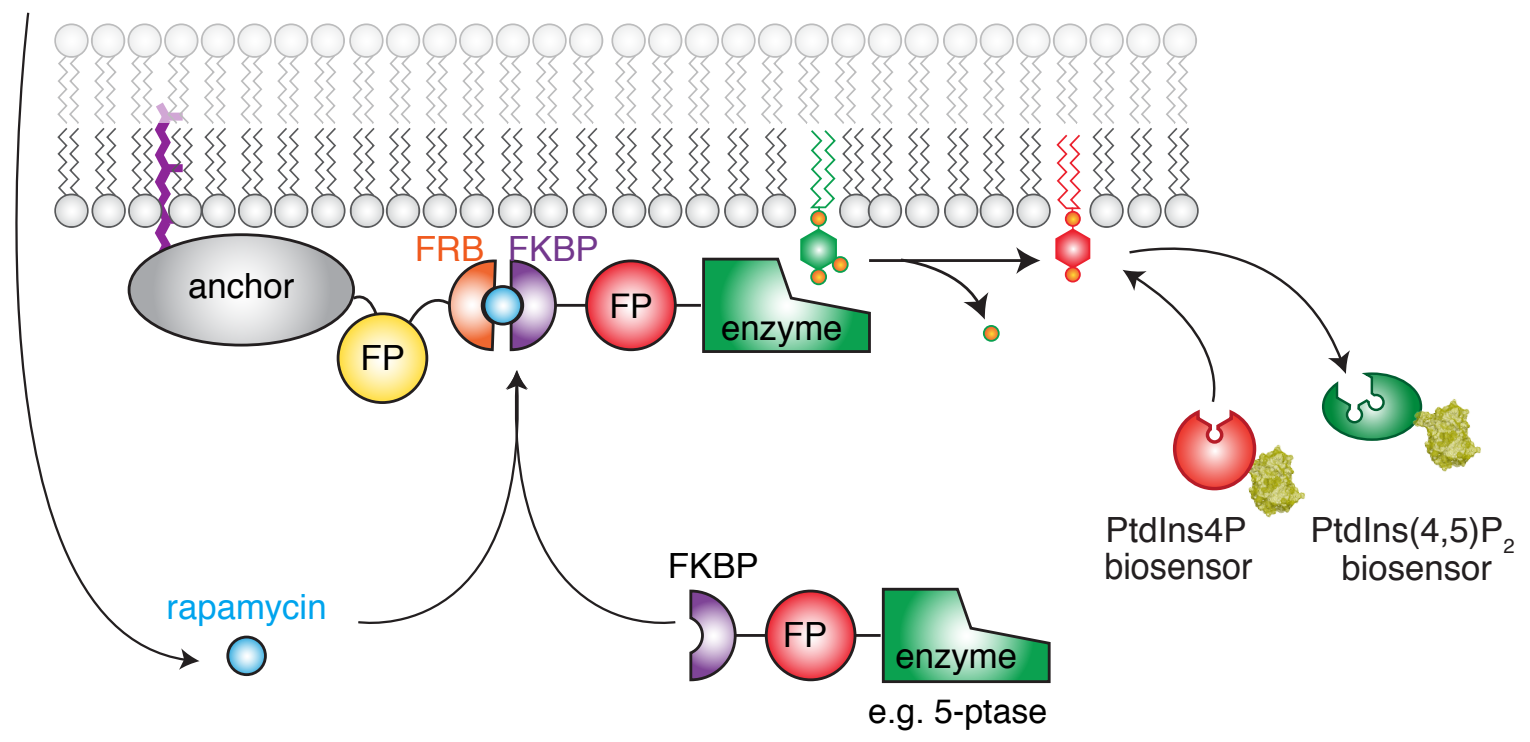

(C) Self-localizing ligand-induced protein translocation (SLIPT) system
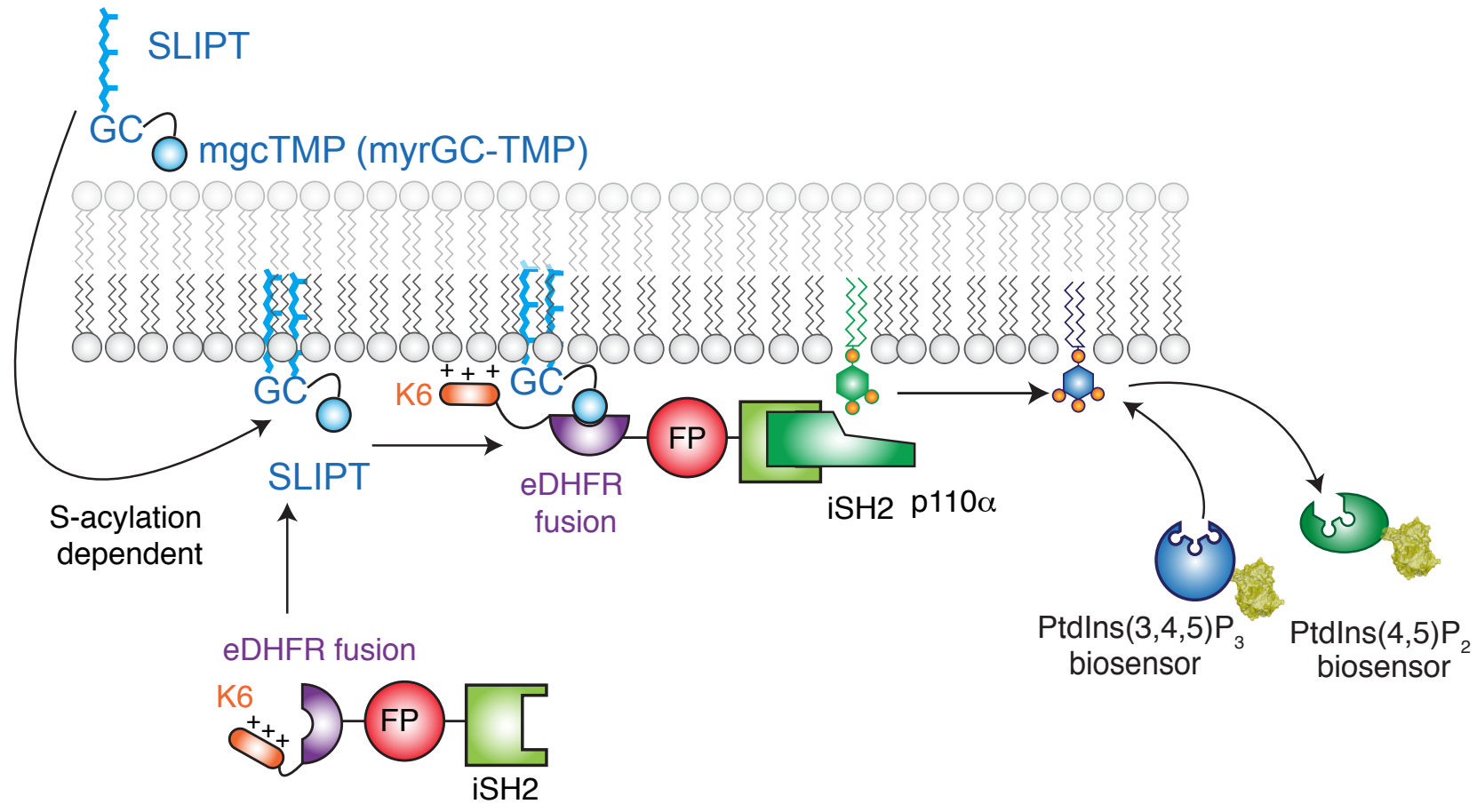
658 Figure 4. Chemogenetic perturbation of PI levels. A) Example showing the fast consumption 659 of PtdIns $(4,5) \mathrm{P}_{2}$ levels via $\mathrm{G}$ receptor-coupled signaling and activation of PLCs. Some cells 660 naturally respond to acetylcholine or oxotremorine $\mathrm{M}(\mathrm{Oxo}-\mathrm{M})$, but other do not. It is possible 661 in that case to express the gene encoding the receptor and $G$ protein in order to render cells 662 responsive to these signals. B) Example showing the principle of rapamycin-induced 663 translocation system based on the conditional FRB-FKBP dimerization. The key to this system 664 is that the catalytic domain of most PI-related enzymes is active at very low levels when they 665 are in the cytosol (i.e. no close contact between the enzyme and its substrate) but they become 666 quickly activated once associated with membranes. C) Example of SLIPT system based on 667 Nakamura et al., (2020). The ligand consists of TMP, a ligand of eDHFR from Escherichia coli, 668 connected to the membrane permeable lipopetide myristoyl-Gly-Cys (myrGC). Once inside the 669 cell, the ligand is inserted in the Golgi membrane, where the cysteine is S-acylated (e.g. 670 palmitoylation) and is then exported to the plasma membrane. However, a fraction remains in 671 the Golgi, likely as unpalmitoylated peptides. In membranes, TMP recruits the eDHFR672 containing protein. Because the eDHFR-fusion protein also contains a polybasic hexalysine 673 peptide (K6), it is stabilized at the plasma membrane owing to its high electronegative 674 properties compared to the Golgi. In this example, the authors fused K6-eDHFR with the SH2 675 domain of $\mathrm{p} 85$ (iSH2), which recruits the catalytically active PI3K subunit $\mathrm{p} 110 \alpha$ and produces $676 \operatorname{PtdIns}(3,4,5) \mathrm{P}_{3}$ from $\operatorname{PtdIns}(4,5) \mathrm{P}_{2}$.

677 Key legend: PtdIns4P, phosphatidylinositol-4-phosphate; PtdIns(4,5) $\mathrm{P}_{2}$, phosphatidylinositol678 (4,5)-bisphosphate; PtdIns(3,4,5) $\mathrm{P}_{2}$, phosphatidylinositol-(3,4,5)-triphosphate; DAG, 679 diacylglycerol; Ins $(1,4,5) \mathrm{P}_{3}$; inositol-(1,3,5)-triphosphate; M1R, muscarinic acetylcholine 680 receptor $\mathrm{M} 1$; Oxo-M, oxotremorine $\mathrm{M}$; G, guanine nucleotide-binding protein; PLC, 681 phospholipase C; FP, fluorescent protein; FKBP, FK506 binding protein; FRB, FKBP682 rapamycin-binding; 5-ptase, inositol 5-phosphatase; SLIPT, self-localizing ligand-induced 683 protein translocation; TMP, trimethoprim; eDHFR, Escherichia coli dihydrofolate reductase; 684 K6, hexalysine peptide; SH2, Src Homology domain2; iSH2, SH2 domain of p85.

685 
(B) Blue-light inducible two-components relocalization system (e.g. CRY2, iLID, Magnets)

CRY2-based system

anchor

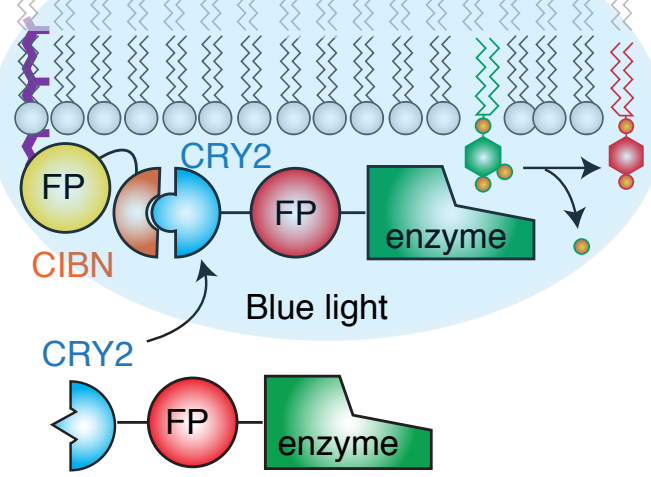

iLID system (LOV-based)

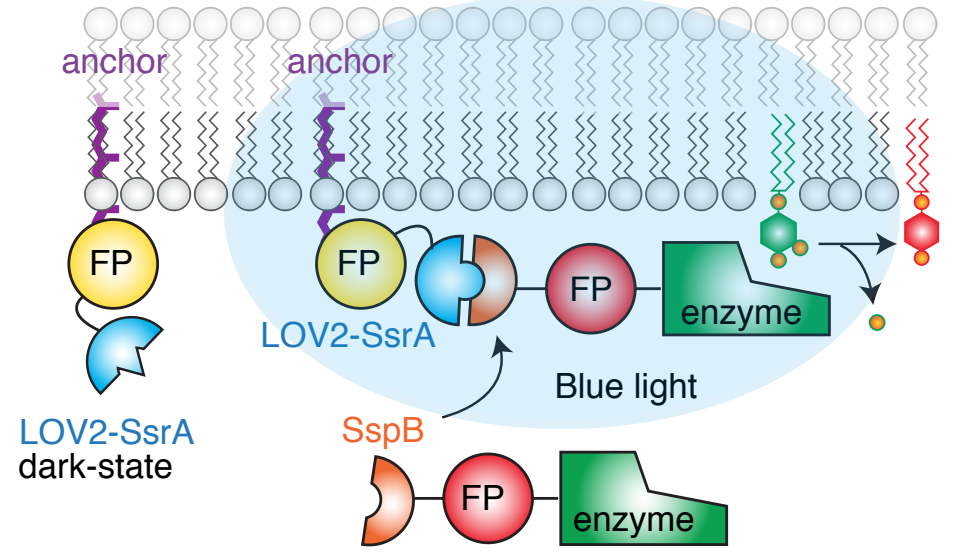

dark-state

Magnets system (LOV ${ }^{\text {ivid-based) }}$

anchor 313anchor?

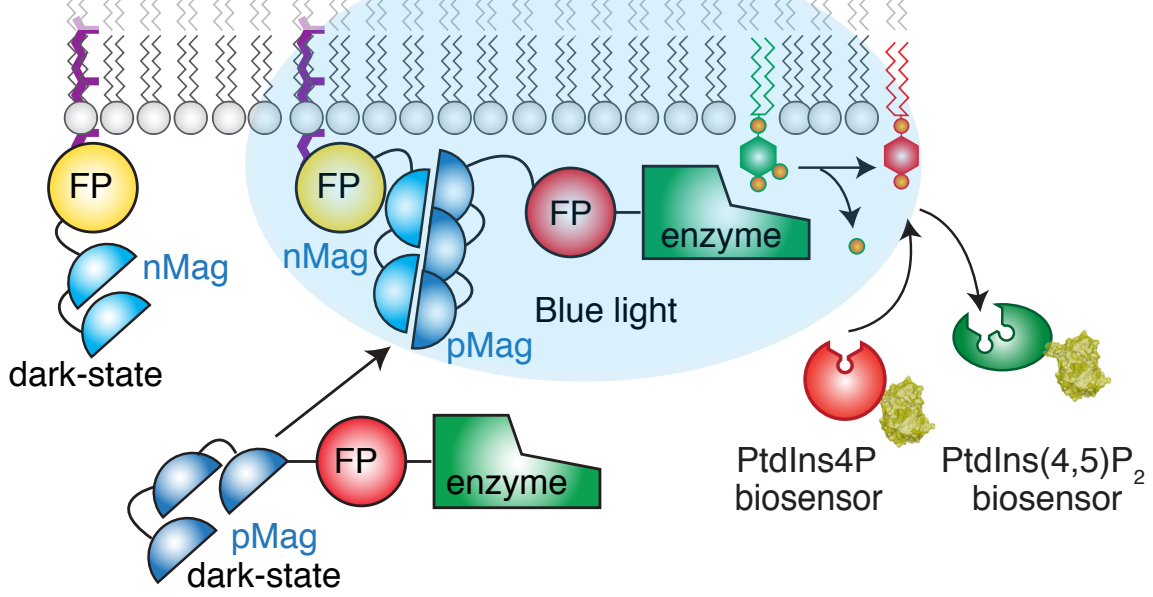

(C) Red/Fad Red-light switch relocalization system (e.g. phytochrome B)

Far-Red light (OFF)

phyB-based system

Red light (ON)

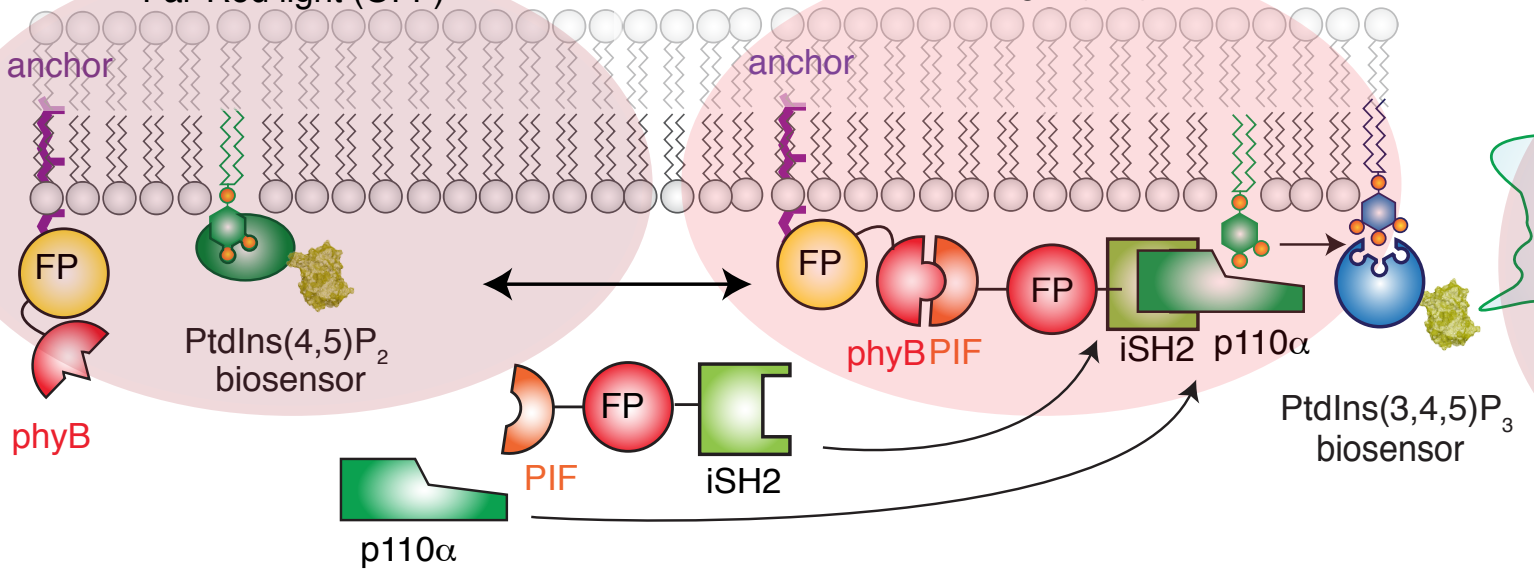

localized PI conversion

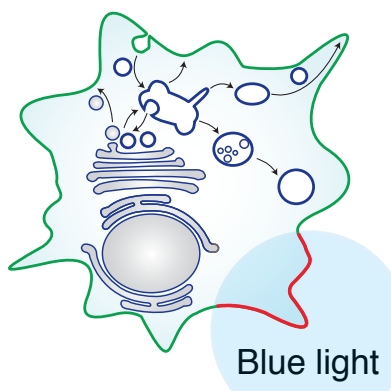

localized \& reversible $\mathrm{PI}$ conversion

Far-Red light
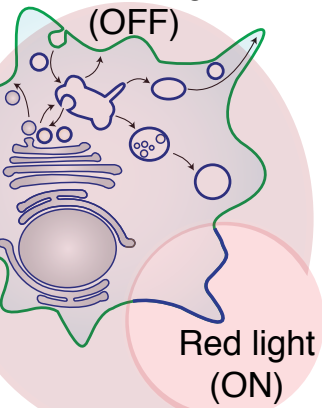

(A) Blue light-inducible single-component relocalization system (e.g. optoPB)

optoPB system (LOV-based)

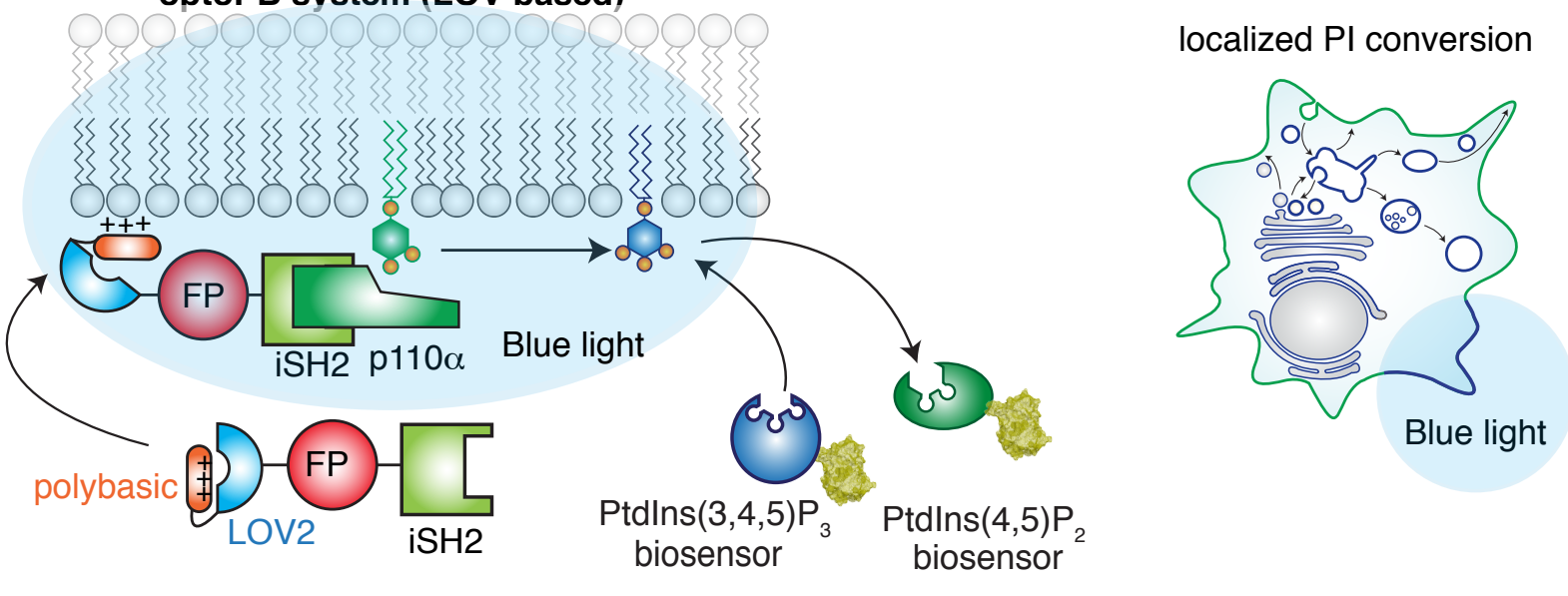


687 Figure 5. Optogenetics perturbation of PI levels. A) Examples showing three different blue-

688 light inducible translocation strategy based on the CRY2/CIBN, iLID and Magnet systems. B)

689 Example based on Toettcher et al., (2011), showing the phyB-based toggle switch, in which red

690 light triggers the recruitment of a PIF-containing chimeric protein (here PIF is fused with iSH2)

691 to the plasma membrane and far red light induces the release of the PIF-containing protein. C)

692 optoPB is a single component optogenetic tool that allows with a single protein to recruit a

693 domain of interest to the plasma membrane upon blue light illumination. In this example, based

694 on He et al., (2017), the authors fused optoPB with the SH2 domain of p85 (iSH2), which

695 recruits the catalytically active PI3K subunit $\mathrm{p} 110 \alpha$ and produces PtdIns $(3,4,5) \mathrm{P}_{3}$ from

696 PtdIns $(4,5) \mathrm{P}_{2}$.

697 Key legend: PI, phosphoinositide; PtdIns4P, phosphatidylinositol-4-phosphate; PtdIns(4,5) $\mathrm{P}_{2}$,

698 phosphatidylinositol-(4,5)-bisphosphate; $\operatorname{PtdIns}(3,4,5) \mathrm{P}_{2}, \quad$ phosphatidylinositol-(3,4,5)-

699 triphosphate; FP, fluorescent protein; CRY, cryptochrome; LOV, Light-oxygen-voltage-

700 sensing; iLID, improved light-induced dimerization; CIBN, N-terminal region of CIB1; CIB1,

701 Cryptochrome-interacting basic-helix-loop-helix1; SspB, 13-kD adaptor protein from E. coli;

702 SsrA, 7-residue peptide that binds SspB; Magnet, engineered Neurospora crassa Vivid

703 photoreceptor, which comprises an N-terminal Ncap domain responsible for homodimerization

704 and a C-terminal light-oxygen-voltage-sensing (LOV) domain, nMag, negative Magnet; pMag,

705 positive Magnet; phyB, phytochromeB; PIF, phytochrome interacting factor; SH2, Src

706 Homology domain2; iSH2, SH2 domain of p85. 